\title{
Формально-языковая модель стиха и регулятивов его метра для автоматизированной метрической идентификации
}

\author{
В.Н. Бойков ${ }^{1}$, М.С. Каряева ${ }^{1}$, И.А. Пильщиков ${ }^{2,3}$ \\ ${ }^{1}$ Ярославский государственный университет, ${ }^{2}$ Московский государственный университет, \\ ${ }^{3}$ Таллиннский университет \\ boykov_bh@bk.ru, mari.karyaeva@gmail.com, pilshch@yandex.ru
}

\section{Аннотация}

Рассматривается формально-языковая модель слогового представления стихового текста поэтического произведения и регулятивов его метра, определенных параметрами некоторых сегментов строки.

Выделены метро-ритмические сегменты, конституирующие как метр, так и ритм стиха, для чего привлечено определение метра русского стиха, опору которому составили такие измеримые сегменты стиха, как фонетичское слово и метрический период с междуиктовым интервалом.

Определены значения регулятивов для репертуара метров русского стиха, в том числе при таких явлениях, как наличие сверхсхемного и пропуск схемного ударения. Описаны процедуры автоматической идентификации метра стихового текста.

Ключевые слова: стих, сегментация стиха, слоговой объем сегмента стиха, междуиктовый интервал, метрообразующий период стиха, метр стиха, регулятивы стихового метра.

Библиографическая ссылка: Бойков В.Н., Каряева М.С., Пильщиков И.А. Формально-языковая модель стиха и регулятивов его метра для автоматизированной метрической идентификации // Компьютерная лингвистика и вычислительные онтологии. Выпуск 3 (Труды XXII Международной объединенной научной конференции «Интернет и современное общество», IMS-2019, Санкт-Петербург, 19 22 июня 2019 г. Сборник научных трудов). - СПб: Университет ИТМО, 2019. С. 124 -141. DOI: 10.17586/2541-9781-2019-3-124-141

\section{1. Введение}

Задача автоматической идентификации метра стихового текста поэтического произведения, несмотря на множество предложенных вариантов ее решения (например: [1], [3], [5], [11], [18], [19]), не теряет своей актуальности в силу того, что остается много вопросов, связанных с определением стиха и стихового метра.

В современной стиховедческой теории принято дескриптивное (неконструктивное) определение стиха, сформулированное М.Л. Гаспаровым: «Стих - это речь, расчлененная на относительно короткие отрезки, строки, соотносимые и соизмеримые между собой» [8], - где соотносимость и соизмеримость - свойства не совсем конструктивные.

В стиховом тексте реализуется метро-ритмическое единство, где под стиховым метром подразумевается схема регулярного чередования сильных (ударных) и слабых (безударных) мест в соотносимых и соизмеримых строках, и правила соответствия ударений реальных ритмических сегментов, составляющих стих, элементам этой схемы.

Законы, по которым это метро-ритмическое единство осуществляется, изучались в прошлом [7], [8], [16] и продолжают изучаться в наше время [12-15], [18]. 
Репертуар стиховых метров и их видов (размеров) практически перечислим, но потенциально неисчерпаем, и тем более при вариациях метро-ритмических схем стиха. Поэтому задача идентификации метра стихового текста сводится к конструктивному построению соответствия метро-ритмическому представлению стиха метрической схемы из репертуара стиховых метров.

\section{2. Формально-языковая модель русского стиха}

Метро-ритмическая структура стихового текста членится на такие элементарные сегменты как слоги, среди которых различаются ударные и безударные. Известно естественное членение речевого ритма на такие сегменты, как «ритмическая группа», состоящая, «по крайней мере, из одного ударного слога и примыкающих к нему безударных слогов» [17] или «акцентная группа» [2], а также «тактовая группа» и «просодический комплекс» [10]. Акцентно самостоятельная словоформа может присоединять к себе так называемые клитики, не обладающие таким свойством словоформы, и в совокупности представлять собой тактовую группу. В стиховом же тексте аналогичным ритмическим сегментом является «фонетическое слово» [7] («метрическое слово» по Р. Якобсону и «прозодический период» по А. Востокову), объединенное вокруг икта или метрического ударения.

Вместе с тем выделяют и такой ритмический сегмент, как «ударный период» [16] или «метрический период» [13], включающий вместе с иктом «междуиктовый интервал».

С учетом различных метрических отклонений от схемы в том и другом случае можно говорить о «метро-ритмическом сегментах» стихового текста, поскольку при априорно неизвестной метрической структуре нельзя знать, какой из ударных слогов является иктом, а какой несет сверхсхемное ударение, и в каком из безударных слогов скрыт пропуск икта. Это вопрос соответствия метро-ритмической схемы стиха метрической схеме.

Метрическая схема и слоговое представление стихового текста и некоторых его компонентов после лингвостиховедческой разметки могут получить формально-языковое представление [4] и [5].

\section{1. Метрическая схема стиха}

Обозначим через А ударные слоги (икты) в метрической схеме стиха. Фонетическое слово, если в безударных слогах различать предударные (предиктовые) $b_{1} b_{2} \ldots b_{r}$ и заударные (заиктовые) слоги $\mathrm{d}_{1} \mathrm{~d}_{2} \ldots \mathrm{d}_{\mathrm{s}}$, можно представить в виде:

(1) $P_{A}=b_{1} b_{2} \ldots b_{r} A d_{1} d_{2} \ldots d_{s}$,

или в свернутом виде

(2) $P_{A j}=b^{r(j)} A d^{s(j)}$,

где $\mathrm{r}_{(\mathrm{j})}$ и $\mathrm{s}_{(\mathrm{j})}$ слоговая длина соответствующих подцепочек.

Слоговой объем фонетического слова Р:

(3) $\left|\mathrm{P}_{\mathrm{Aj}}\right|=\left(\mathrm{r}_{(\mathrm{j})}+\mathrm{s}_{(\mathrm{j})}+1\right)$,

где $\mathrm{k}$ - число иктов и $\mathrm{j}=1,2, . . \mathrm{k}$.

Схема k-иктовой строки схематически представляется последовательностью $\mathrm{k}$ фонетических слов:

(4) $F_{A}=\prod_{j=1,2, . . k} P_{A i j}=\prod_{j=1,2, . . k} b^{r(j)} A_{j} d^{s(j)}=b^{r(1)} A_{1} d^{s(1)} b^{r(2)} A_{2} d^{s(2)} \ldots b^{r(j)} A_{j} d^{s(j)} \ldots b^{r(k)} A_{k} d^{s(k)}$.

Откуда нетрудно вывести слоговой объем строки:

(5) $\left|\mathrm{F}_{\mathrm{A}}\right|=\left(\mathrm{k}+\sum_{\mathrm{j}=1,2, \ldots \mathrm{k}}\left(\mathrm{r}_{(\mathrm{j})}+\mathrm{s}_{(\mathrm{j})}\right)\right)$.

Однако подобное же представление имеет и прозаический текст, разбитый на сегменты из $\mathrm{k}$ слов, поэтому имеет смысл выделить в схеме стиха метрический период $\mathrm{U}_{\mathrm{Aj}}$ :

(6) $\mathrm{U}_{\mathrm{Aj}}=\mathrm{A}_{\mathrm{j}} \mathrm{d}^{\mathrm{s}(\mathrm{j})} \mathrm{b}^{\mathrm{r}(\mathrm{j}+1)}=\mathrm{A}_{\mathrm{j}} \mathrm{V}_{\mathrm{Aj}}$,

так называемую стопу со слоговым объемом

(7) $\left|U_{\mathrm{Aj}}\right|=\left(1+\mathrm{s}_{(\mathrm{j})}+\mathrm{r}_{(\mathrm{j}+1)}\right)$. 
Здесь примыкающий к икту $\mathrm{A}_{i}$ междуиктовый интервал

(8) $\mathrm{V}_{\mathrm{Aj}}=\mathrm{d}^{\mathrm{s}(\mathrm{j})} \mathrm{b}^{\mathrm{r}(\mathrm{j}+1)}$

имеет слоговой объем

(9) $\left|\mathrm{V}_{\mathrm{Aj}}\right|=\left(\mathrm{s}_{(\mathrm{j})}+\mathrm{r}_{(\mathrm{j}+1)}\right)$

при вариативности объема междуиктовых интервалов

(10) $\mathrm{v}=\left(\left|\mathrm{V}_{\mathrm{A}}\right|_{\max }-\left|\mathrm{V}_{\mathrm{A}}\right|_{\min }\right)=\left(\left|\mathrm{U}_{\mathrm{A}}\right|_{\max }-\left|\mathrm{U}_{\mathrm{Aj}}\right|_{\min }\right)$,

так что $\left|\mathrm{V}_{\mathrm{A}}\right|_{\min } \leq\left|\mathrm{V}_{\mathrm{Aj}}\right| \leq\left|\mathrm{V}_{\mathrm{A}}\right|_{\max }$ и $\left|\mathrm{U}_{\mathrm{A}}\right|_{\max } \leq\left|\mathrm{U}_{\mathrm{Aj}}\right| \leq\left|\mathrm{U}_{\mathrm{Aj}}\right|_{\min }$.

Тогда схема $\mathrm{k}$-иктовой строки может быть представлена последовательностью (k-1) таких периодов или стоп:

(11) $\mathrm{F}_{\mathrm{A}}=\mathrm{b}^{\mathrm{r}(1)}\left(\prod_{\mathrm{j}=1,2, . .(\mathrm{k}-1)} \mathrm{S}_{\mathrm{Aj}}\right) \mathrm{A}_{\mathrm{k}} \mathrm{d}^{\mathrm{s}(\mathrm{k})}=\mathrm{b}^{\mathrm{r}(1)}\left(\prod_{\mathrm{j}=1,2, . .(\mathrm{k}-1)} \mathrm{A}_{\mathrm{j}} \mathrm{d}^{\mathrm{s}(\mathrm{j})} \mathrm{b}^{\mathrm{r}(\mathrm{j}+1)}\right) \mathrm{A}_{\mathrm{k}} \mathrm{d}^{\mathrm{s}(\mathrm{k})}$,

где, кроме того, начальная предударная подцепочка $\mathrm{b}^{\mathrm{r}(1)}$ представляет собой анакрусу. $\mathrm{a}$ заключительная $\mathrm{A}_{\mathrm{k}} \mathrm{d}^{\mathrm{s}(\mathrm{k})}$ эпикруза состоит из последнего икта $\mathrm{A}_{\mathrm{k}}$ и клаузулы - конечной заударной подцепочки $\mathrm{d}^{\mathrm{s}(\mathrm{k})}$.

Слоговой объем строки представляется через сумму объемов стоп $\left|\mathrm{U}_{\mathrm{Ai}}\right|$ :

(12) $\left|\mathrm{F}_{\mathrm{A}}\right|=\left(\mathrm{r}_{(1)}+\mathrm{s}_{(\mathrm{k})}+1+\sum_{\mathrm{j}=1,2, \ldots(\mathrm{k}-1)}\left|\mathrm{U}_{\mathrm{Aj}}\right|=\left(\mathrm{r}_{(1)}+\mathrm{s}_{(\mathrm{k})}+1+\sum_{\mathrm{j}=1,2, \ldots(\mathrm{k}-1)}\left(1+\mathrm{s}_{(\mathrm{j})}+\mathrm{r}_{(\mathrm{j}+1)}\right)\right)\right.$,

или объемов междуиктовых интервалов $\left|\mathrm{V}_{\mathrm{j}}\right|$ :

$\left|\mathrm{F}_{\mathrm{A}}\right|=\left(\mathrm{r}_{(1)}+\mathrm{s}_{(\mathrm{k})}+\mathrm{k}+\sum_{\mathrm{j}=1,2, \ldots(\mathrm{k}-1)}\left|\mathrm{V}_{\mathrm{Aj}}\right|\right)=\left(\mathrm{r}_{(1)}+\mathrm{s}_{(\mathrm{k})}+\mathrm{k}+\sum_{\mathrm{j}=1,2, \ldots(\mathrm{k}-1)}\left(\mathrm{s}_{(\mathrm{j})}+\mathrm{r}_{(\mathrm{j}+1)}\right)\right)$.

Важно выделение также следующих сегментов стиха и их объемных параметров.

Основу стиха без клаузулы $\mathrm{d}^{\mathrm{s}(\mathrm{k})}$ составляет согласно схемам (4) и (11) цепочка (k-1) фонетических слов $\mathrm{P}_{\mathrm{Aj}}=\mathrm{b}^{\mathrm{r}(\mathrm{j})} \mathrm{Ad}^{\mathrm{s}(\mathrm{j})}$ без заударной части последнего $\mathrm{b}^{\mathrm{r}(\mathrm{k})} \mathrm{A}_{\mathrm{k}}$ или цепочка анакрусы $\mathrm{b}^{\mathrm{r}(1)}$, метрических периодов (стоп) $\mathrm{U}_{\mathrm{Aj}}=\mathrm{A}_{\mathrm{i}} \mathrm{d}^{\mathrm{s}(\mathrm{j})} \mathrm{b}^{\mathrm{r}(\mathrm{j}+1)}$ и последнего икта $\mathrm{A}_{\mathrm{k}}$ :

(13) $\mathrm{W}_{\mathrm{A}}=\left(\prod_{\mathrm{j}=1,2, . .(\mathrm{k}-1)} \mathrm{b}^{\mathrm{r}(\mathrm{j})} \mathrm{Ad}^{\mathrm{s}(\mathrm{j})}\right) \mathrm{b}^{\mathrm{r}(\mathrm{k})} \mathrm{A}_{\mathrm{k}}=\mathrm{b}^{\mathrm{r}(1)}\left(\prod_{\mathrm{j}=1,2, . .(\mathrm{k}-1)} \mathrm{A}_{\mathrm{i}} \mathrm{d}^{\mathrm{s}(\mathrm{j})} \mathrm{b}^{\mathrm{r}(\mathrm{j}+1)}\right) \mathrm{A}_{\mathrm{k}}$ со слоговым объемом

$\left|\mathrm{W}_{\mathrm{A}}\right|=\left(\mathrm{k}+\mathrm{r}_{(\mathrm{k})}+\sum_{\mathrm{j}=1,2, \ldots(\mathrm{k}-1)}\left(\mathrm{r}_{(\mathrm{j})}+\mathrm{s}_{(\mathrm{j})}\right)\right)=\left(\mathrm{r}_{(1)}+\mathrm{k}+\sum_{\mathrm{j}=1,2, \ldots(\mathrm{k}-1)}\left(\mathrm{s}_{(\mathrm{j})}+\mathrm{r}_{(\mathrm{j}+1)}\right)\right)$.

Подцепочки фонетических слов от начала стиха до словораздела после і-го икта, $\mathrm{i}=1,2, \ldots(\mathrm{k}-1)$ :

(14) $\mathrm{F}_{\mathrm{Ai}}=\mathrm{b}^{\mathrm{r}(1)} \mathrm{A}_{1} \mathrm{~d}^{\mathrm{s}(1)} \mathrm{b}^{\mathrm{r}(2)} \mathrm{A}_{2} \mathrm{~d}^{\mathrm{s}(2)} \ldots \mathrm{b}^{\mathrm{r}(\mathrm{i})} \mathrm{A}_{\mathrm{i}} \mathrm{d}^{\mathrm{s}(\mathrm{i})}$ со слоговым объемом словораздела

$\left|\mathrm{F}_{\mathrm{Ai}}\right|=\sum_{\mathrm{g}=1,2, \ldots \mathrm{i}}\left|\mathrm{P}_{\mathrm{Ag}}\right|=\sum_{\mathrm{g}=1,2, \ldots \mathrm{i}}\left(\mathrm{r}_{(\mathrm{g})}+\mathrm{S}_{(\mathrm{g})}+1\right)=\left(\mathrm{i}+\sum_{\mathrm{g}=1,2, \ldots \mathrm{i}}\left(\mathrm{r}_{(\mathrm{g})}+\mathrm{S}_{(\mathrm{g})}\right)\right)$.

\section{2. Метро-ритмическое представление стиха}

По аналогии с метрической сегментацией рассмотрим сегментацию слогового представления реального стихового текста.

Метро-ритмическое слово реального стихового текста можно представить цепочками безударных слогов с, примыкающих к ударному слогу C:

(15) $\mathrm{P}_{\mathrm{C}}=\mathrm{c}_{1} \mathrm{c}_{2} \ldots \mathrm{c}_{\mathrm{q}} \mathrm{Cc}_{1} \mathrm{c}_{2} \ldots \mathrm{c}_{\mathrm{h}}$,

или в свернутом виде

(16) $\mathrm{P}_{\mathrm{Cj}}=\mathrm{c}^{\mathrm{q}(\mathrm{j})} \mathrm{C}_{\mathrm{j}} \mathrm{c}^{\mathrm{h}(\mathrm{j})}$,

со слоговым объемом

(17) ) $\left|\mathrm{P}_{\mathrm{Cj}}\right|=\left(\mathrm{q}_{\mathrm{j} 0}+\mathrm{h}_{(\mathrm{j})}+1\right)$,

где $\mathrm{z}$ - число ударных слогов и $\mathrm{j}=1,2, . . \mathrm{z}$.

Тогда z-ударная строка стихового текста представляется последовательностью z метроритмических слов:

(18) $\mathrm{F}_{\mathrm{C}}=\mathrm{c}^{\mathrm{q}(1)} \mathrm{C}_{1} \mathrm{c}^{\mathrm{h}(1)} \mathrm{c}^{\mathrm{q}(2)} \mathrm{C}_{2} \mathrm{c}^{\mathrm{h}(2)} \ldots \mathrm{c}^{\mathrm{q}(\mathrm{j})} \mathrm{C}_{\mathrm{j}} \mathrm{c}^{\mathrm{h}(\mathrm{j}))} \ldots \mathrm{c}^{\mathrm{q}(\mathrm{z})} \mathrm{C}_{\mathrm{z}} \mathrm{c}^{\mathrm{h}(\mathrm{z})}$,

со слоговой объемом:

(19) $\left|\mathrm{F}_{\mathrm{C}}\right|=\left(\mathrm{z}+\sum_{\mathrm{j}=1,2, \ldots \mathrm{z}}\left(\mathrm{q}_{(\mathrm{j})}+\mathrm{h}_{(\mathrm{j})}\right)\right)$.

Здесь начальная предударная подцепочка $\mathrm{c}^{\mathrm{q}(1)}$ может: 1) представлять собой анакрусу, 2) может содержать в себе анакрузу с пропуском ударения на икте, 3) может содержаться в анакрузе как сверхсхемное ударение.

Заключительная подцепочка $\mathrm{C}_{\mathrm{z}} \mathrm{c}^{\mathrm{h}(\mathrm{z})}$ скорее всего совпадает со схемной эпикрузой $\mathrm{A}_{\mathrm{z}} \mathrm{c}^{\mathrm{h}(\mathrm{z})}$, поскольку, по «правилу Томашевского - Якобсона», пропуск ударения на последнем икте при реализации метрической схемы запрещен и клаузула сверхсхемное ударение обычно не принимает, поэтому можно считать, что последний ударный слог в метро-ритмической схеме приходится на икт $-\mathrm{C}_{\mathrm{z}} \approx \mathrm{A}$, то есть 


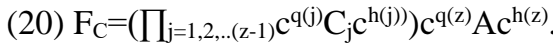

Можно выделить междуакцентные интервалы

(21) $\mathrm{V}_{\mathrm{Cj}}=\mathrm{c}^{\mathrm{h}(\mathrm{j})} \mathrm{c}^{\mathrm{q}(\mathrm{j}+1)}$

со слоговым объемом

(22) $\left|\mathrm{V}_{\mathrm{Cj}}\right|=\left(\mathrm{h}_{(\mathrm{j})}+\mathrm{q}_{(\mathrm{j}+1)}\right)$,

и определить среди них $\left|V_{C}\right|_{\min } \leq\left|V_{C j}\right| \leq\left|V_{C}\right|_{\max }$. Однако здесь разность $\left(\left|V_{C}\right|_{\max }-\left|V_{C}\right|_{\min }\right)$ не определяет заданный показатель $\mathrm{v}=\left(\left|\mathrm{V}_{\mathrm{A}}\right| \max -\left|\mathrm{V}_{\mathrm{A}}\right|_{\min }\right)$ вариативности объема междуиктовых интервалов, ввиду возможности сверхсхемных ударений и пропусков ударений на иктах.

Основу строки стихового текста, за вычетом клаузулы $\mathrm{c}^{\mathrm{h}(\mathrm{z})}$, составляет цепочка анакрусы $\mathrm{c}^{\mathrm{q}(1)}$, ударных слогов с междуакцентными интервалами $\mathrm{Cc}^{\mathrm{h}(\mathrm{j})} \mathrm{c}^{\mathrm{q}(\mathrm{j}+1)}$ и последнего икта $\mathrm{A}_{\mathrm{z}}$ :

(23) $\mathrm{W}_{\mathrm{C}}=\mathrm{c}^{\mathrm{q}(1)}\left(\prod_{\mathrm{j}=1,2, . . \mathrm{z}-1)} \mathrm{C}_{\mathrm{j}} \mathrm{c}^{\mathrm{h}(\mathrm{j})} \mathrm{c}^{\mathrm{q}(\mathrm{j}+1)}\right) \mathrm{A}_{\mathrm{z}}$ со слоговым объемом

$\left|\mathrm{W}_{\mathrm{C}}\right|=\left(\mathrm{q}_{(1)}+\mathrm{z}+\sum_{\mathrm{j}=1,2, \ldots(\mathrm{z}-1)}\left(\mathrm{h}_{(\mathrm{j})}+\mathrm{q}_{(\mathrm{j}+1)}\right)\right)$,

при этом следует считать, что $\left|\mathrm{W}_{\mathrm{C}}\right|=\left|\mathrm{W}_{\mathrm{A}}\right|$.

Подцепочки метро-ритмических слов от начала стиха до словораздела после ј-го ударного слога, $\mathrm{j}=1,2, \ldots(\mathrm{z}-1)$ :

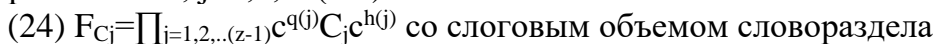

$\left|\mathrm{F}_{\mathrm{Ci}}\right|=\left(\mathrm{j}+\sum_{\mathrm{i}=1,2, \ldots(\mathrm{z}-1)}\left(\mathrm{q}_{(\mathrm{i})}+\mathrm{h}_{(\mathrm{i})}\right)\right)$.

\section{3. Регулятивы, определение метра стиха и выбор системы стихосложения}

Комплекс регулятивов, определяющих метрическую схему стиха, представляют слоговые объемы выделенных сегментов в описанных выше формулах: (3) $\left|\mathrm{P}_{\text {Aj }}\right|=\left(\mathrm{r}_{(\mathrm{j})}+\mathrm{s}_{(\mathrm{j})}+1\right)$, (5) и (12) $\quad\left|\mathrm{F}_{\mathrm{A}}\right|=\left(\mathrm{k}+\sum_{\mathrm{j}=1,2, \ldots \mathrm{k}}\left(\mathrm{r}_{(\mathrm{j})}+\mathrm{s}_{(\mathrm{j})}\right)\right) \quad$ и $\quad\left|\mathrm{F}_{\mathrm{A}}\right|=\left(\mathrm{r}_{(1)}+\mathrm{s}_{(\mathrm{k})}+\mathrm{k}+\sum_{\mathrm{j}=1,2, \ldots(\mathrm{k}-1)}\left(\mathrm{s}_{(\mathrm{j})}+\mathrm{r}_{(\mathrm{j}+1)}\right)\right)$ $\left|\mathrm{U}_{\mathrm{Aj}}\right|=\left(1+\mathrm{s}_{(\mathrm{j})}+\mathrm{r}_{(\mathrm{j}+1)}\right), \quad$ (9) $\quad\left|\mathrm{V}_{\mathrm{Aj}}\right|=\left(\mathrm{s}_{(\mathrm{j})}+\mathrm{r}_{(\mathrm{j}+1)}\right), \quad$ (10) $\quad \mathrm{V}=\left(\left|\mathrm{V}_{\mathrm{A}}\right| \max -\left|\mathrm{V}_{\mathrm{A}}\right|_{\min }\right)=\left(\left|\mathrm{U}_{\mathrm{A}}\right|_{\max }-\left|\mathrm{U}_{\mathrm{A}}\right|_{\min }\right)$, $\left|\mathrm{V}_{\mathrm{A}}\right|_{\min } \leq\left|\mathrm{V}_{\mathrm{Aj}}\right| \leq\left|\mathrm{V}_{\mathrm{A}}\right|_{\max } \quad$ и $\quad\left|\mathrm{U}_{\mathrm{A}}\right|_{\max } \leq\left|\mathrm{U}_{\mathrm{Aj}}\right| \leq\left|\mathrm{U}_{\mathrm{Aj}}\right|_{\min }, \quad$ (13) $\quad\left|\mathrm{W}_{\mathrm{A}}\right|=\left(\mathrm{r}_{(1)}+\mathrm{k}+\sum_{\mathrm{j}=1,2, \ldots(\mathrm{k}-1)}\left(\mathrm{s}_{(\mathrm{j})}+\mathrm{r}_{(\mathrm{j}+1)}\right)\right)$, (14) $\left|\mathrm{F}_{\mathrm{Ai}}\right|=\left(\mathrm{r}_{(1)}+\mathrm{s}_{(\mathrm{i})}+\mathrm{i}+\sum_{\mathrm{j}=1,2, \ldots \mathrm{i}}\left(\mathrm{s}_{(\mathrm{g})}+\mathrm{r}_{(\mathrm{g}+1)}\right)\right)$ и заданное число иктов $\mathrm{k}$.

Какие-то из этих регулятивов могут быть определены из слоговых объемов выделенных сегментов метро-ритмического представления стихового текста по формулам: (17) $\left|\mathrm{P}_{\mathrm{Cj}}\right|=\left(\mathrm{q}_{(\mathrm{j})}+\mathrm{h}_{(\mathrm{j})}+1\right),(19)\left|\mathrm{F}_{\mathrm{C}}\right|=\left(\mathrm{z}+\sum_{\mathrm{j}=1,2, \ldots \mathrm{z}}\left(\mathrm{q}_{(\mathrm{j})}+\mathrm{h}_{(\mathrm{j})}\right)\right),(22)\left|\mathrm{V}_{\mathrm{Cj}}\right|=\left(\mathrm{h}_{(\mathrm{j})}+\mathrm{q}_{(\mathrm{j}+1)}\right),\left|\mathrm{V}_{\mathrm{C}}\right|_{\min } \leq\left|\mathrm{V}_{\mathrm{C} j}\right| \leq\left|\mathrm{V}_{\mathrm{C}}\right|_{\max }$, (23) $\left|\mathrm{W}_{\mathrm{C}}\right|=\left(\mathrm{z}-1+\sum_{\mathrm{j}=1,2, \ldots(\mathrm{z}-1)}\left(\mathrm{h}_{(\mathrm{j})}+\mathrm{q}_{(\mathrm{j}+1)}\right)\right)$ и по числу размеченных акцентов $\mathrm{z}$, если учесть, что в урегулированных метро-ритмических формах стиха возможны сверхсхемные ударения (по «правилу Томашевского - Якобсона» ударения попадающих в междуиктовый интервал слов, слоговая длина которых $\leq\left|\mathrm{V}_{\mathrm{Ai}}\right|$ ) и пропуски схемных ударений на иктах (кроме последнего - это действующее в русском стихе правило последней ударной константы).

Нетрудно видеть, что в метрической схеме стиха для всех регулятивов, исключая объемы фонетических слов (3) $\left|\mathrm{P}_{\mathrm{Aj}}\right|=\left(\mathrm{r}_{(\mathrm{j})}+\mathrm{s}_{(\mathrm{j})}+1\right)$, определяющими являются величины междуиктовых интервалов (9) $\left|\mathrm{V}_{\mathrm{Aj}}\right|=\left(\mathrm{s}_{(\mathrm{j})}+\mathrm{r}_{(\mathrm{j}+1)}\right)$ и, в первую очередь, для метрического периода (7) $\left|\mathrm{U}_{\mathrm{Aj}}\right|=\left(1+\mathrm{s}_{(\mathrm{j})}+\mathrm{r}_{(\mathrm{j}+1)}\right)$ и для показателя вариативности объема междуиктовых интервалов $\mathrm{v}=\left(|\mathrm{V}|_{\max }-|\mathrm{V}|_{\min }\right)$.

Для идентификации метра по аналогам регулятивов, представленных слоговыми объемами выделенных сегментов метро-ритмического представления стихового текста формул (17), (19), (22), (23) необходимо иметь достаточно конструктивное определение стихового метра.

Таковым представляется с опорой на сформулированное в [13] следующее ОПРЕДЕЛЕНИЕ метра русской стихотворной строки:

Метр - это схема расположения сильных мест (иктов) и слабых мест (междуиктовых интервалов) (9) $\left|\mathrm{V}_{\mathrm{Aj}}\right|=\left(\mathrm{s}_{(\mathrm{j})}+\mathrm{r}_{(\mathrm{j}+1)}\right)$, в стихотворной строке, где ударение слова со слоговым объемом, большим величины постоянного или максимального объема междуиктовых интервалов, не может попадать на слабые места и должно попадать на одно из 
покрываемых им сильных мест. Эти правила должны быть применимы ко всем строкам стихового текста.

Нетрудно видеть, что в этом определении метра наряду со слоговыми объемами междуиктовых интервалов важными являются также слоговые объемы фонетических слов (3) $\left|\mathrm{P}_{\mathrm{Aj}}\right|=\left(\mathrm{r}_{(\mathrm{j})}+\mathrm{s}_{(\mathrm{j})}+1\right)$.

Сохраняя обозначение ударных слогов прописными буквами, безударных - строчными, можно выявить в метро-ритмической схеме стиха метрическую основу. В этом случае исходя из формул (18) и (20) следует ударные слоги С, приходящиеся на сильные места (икты), согласно формулам (4) и (11) переобозначить $\mathrm{C} \rightarrow \mathrm{A}$, а приходящиеся на слабые места $-\mathrm{C} \rightarrow \mathrm{B}$ или $\mathrm{C} \rightarrow \mathrm{D}$, как явление сверхсхемных ударений. Безударные слоги формул (18) и (20), приходящиеся на сильные места, следует согласно формулам (4) и (11) переобозначить $\mathrm{c} \rightarrow \mathrm{a}$, как пропуск ударения на икте, а приходящиеся на слабые места $\mathrm{c} \rightarrow \mathrm{b}$ или $\mathrm{c} \rightarrow \mathrm{d}$. Таким образом, в соответствии с заданным определением метра выявление метрической схемы стиха в метро-ритмической сводится к поиску подходящих преобразований $\mathrm{C} \rightarrow \mathrm{A}$ и $\mathrm{c} \rightarrow \mathrm{a}$.

\section{1. Системы стихосложения}

Прежде чем, следуя заданному определению метра, подступаться к процедуре выявления ударных слогов, приходящихся на икты, по слоговым объемам формул (17), (19), (22), (23) в метро-ритмической схеме стиха, необходимо установить, опираясь на определенные регулятивы, к какой системе стихосложения из нижеследующего систематического перечня типов метрики русского стиха относится исследуемый стиховой текст.

1. Метрический стих

Стих, урегулированный по слоговому объему строки $|\mathrm{F}|$, по числу $\mathrm{k}$ ударных слогов на метрообразующих местах (иктах), по слоговому объему междуиктовых интервалов $|\mathrm{V}| \mathrm{c}$ ограничениями на вариативность их объема $\mathrm{v}=(|\mathrm{V}| \mathrm{max}-|\mathrm{V}| \mathrm{min})$.

1.1. Силлабический стих.

Стих с равными слоговыми объемами строк $|\mathrm{F}|$ и с женскими клаузулами, состоящими из одного слога (с или d), для всех строк текста, иногда с цезурой на постоянном для всех строк текста словоразделе.

1.2. Силлабо-тонический стих.

Стих с равными слоговыми объемами основы строк $|\mathrm{W}|$ и с вариативностью междуиктовых интервалов $\mathrm{v}=0$, однако допускающий наличие сверхсхемных ударений и пропуск ударений на иктах.

Стихотворный размер этого типа метрики определяется комбинацией слоговых объемов междуиктового интервала $|\mathrm{V}| \mathrm{w}=\mathrm{w}, \mathrm{w}=0,1,2,3,4,5$, и анакрусы $\mathrm{r}(1)=0,1, \ldots \mathrm{w}$.

Теоретическое число таких комбинаций $21=1(\mathrm{w}=0)+2(\mathrm{w}=1)+3(\mathrm{w}=2)+4(\mathrm{w}=3)+5(\mathrm{w}=4)+6(\mathrm{w}=5)$.

1.3. Тонический стих.

Стих с постоянным числом $\mathrm{k}$ ударных иктовых слогов и допускающий наличие сверхсхемных ударений.

1.3.1. Стих с заданной вариативностью междуиктовых интервалов v и допускающий пропуск ударений на иктах.

1.3.1.1. Дольник - тонический стих с вариативностью междуиктовых интервалов $\mathrm{v}=1$.

1.3.1.2. Тактовик - тонический стих с вариативностью междуиктовых интервалов v=2.

1.3.2. Стих с ограниченной сверху в речевой практике русского языка вариативностью междуиктовых интервалов $\mathrm{v} \leq 8$ и не допускающий пропуск ударений на иктах.

1.3.2.1. Ударник (акцентный стих) - стих с ограниченной снизу вариативностью междуиктовых интервалов $3 \leq \mathrm{v}$.

1.3.3.2. Строчный логаэд - стих с равными слоговыми объемами основы строк $|\mathrm{W}|$ и с совпадением ударений на иктах. 
2. Дисметрический (свободный) стих.

Стих, не урегулированный по метрическим параметрам, с произвольными регулятивами: слоговым объемом строки, числом ударных слогов и вариативностью междуиктовых интервалов $\mathrm{v} \leq 8$.

2.1. Верлибр - свободный стих, с неурегулированной клаузулой.

2.2. Раешный стих - свободный стих, с рифмованной клаузулой.

Для выявления соотносимых и соизмеримых строк необходимо предварительно осуществить пошаговые процедуры по ряду регулятивов с указанием возможных типов метрики из приведенного выше систематического перечня.

Шаг 1. Произвести согласно [6] поиск рифмующихся строк, задающих строфику стихового текста:

Шаг 1.1. если все строки рифмованного текста имеют равный слоговой объем основ $|\mathrm{W}|=$ const и равный объем клаузул $\mathrm{h}(\mathrm{z})=1$, тогда альтернативными являются силлабический стих, силлабо-тонический стих и строчный логаэд;

Шаг 1.2. в противном случае для рифмованных строк с равными слоговыми объемами основ $|\mathrm{W}|$ альтернативными являются силлабо-тонический стих и строчный логаэд;

Шаг 1.3. для рифмованных строк с неравными слоговыми объемами основ $|\mathrm{W}|$ и с равным числом ударных слогов $\mathrm{z}$ альтернативными являются виды тонического стиха с заданной вариативностью междуиктовых интервалов v (без сверхсхемных ударений и без пропуска ударений на иктах);

Шаг 1.4. для рифмованных строк с неравными слоговыми объемами основ $|\mathrm{W}|$ и с неравным числом ударных слогов $\mathrm{z}$ альтернативными являются вольный силлаботонический стих, виды тонического стиха с заданной вариативностью междуиктовых интервалов v (с наличием сверхсхемных ударений и с пропуском ударений на иктах) и раешный стих;

Шаг 2 Для слоговых объемов основ строк $|\mathrm{W}|$ не рифмованного текста независимо от объемов клаузул надо установить:

Шаг 2.1. если все строки не рифмованного текста имеют равный слоговой объем основ $|\mathrm{W}|=\mathrm{const}$, тогда безальтернативен белый силлабо-тонический стих;

Шаг 2.2. в противном случае если строки не рифмованного текста не имеют равного слогового объема основ $|\mathrm{W}| \neq$ const, тогда альтернативными являются вольный белый силлабо-тонический стих и свободный стих.

В этом соотнесении все типы метрики из систематического перечня оказываются охваченными, однако сочетания выделенных регулятивов не являются достаточными для идентификации конкретного метра.

\section{2. Разрешение метрических альтернатив}

Для разрешения альтернатив Шагов 1.1. и 1.2. надо учесть следующее: силлабический стих дополняется таким регулятивом, как при $|\mathrm{W}| \geq 8$ возможная цезура на словоразделе между полустишиями, и не имеет таких регулятивов, как объемы метрической анакрусы $\mathrm{r}(1)$ и междуиктовых интервалов $|\mathrm{VAj}|=(\mathrm{s}(\mathrm{j})+\mathrm{r}(\mathrm{j}+1))$; силлабо-тонический стих дополняется такими регулятивами, как объем метрической анакрусы $\mathrm{r}(1)$ и междуиктовых интервалов $|\mathrm{VAj}|=(\mathrm{s}(\mathrm{j})+\mathrm{r}(\mathrm{j}+1))=\mathrm{const}$; строчный логаэд дополняется такими регулятивами, как объем метрической анакрусы $\mathrm{r}(1)$ и совпадение ударений на иктах соизмеряемых строк, и не имеет таких регулятивов, как объем междуиктовых интервалов $|\mathrm{VAj}|=(\mathrm{s}(\mathrm{j})+\mathrm{r}(\mathrm{j}+1))=\mathrm{const}$.

В первую очередь для разрешения альтернативы между силлабическим и силлаботоническим стихом и строчным логаэдом в тексте со строками одинакового объема и с женской рифмой следует выявить метрическую анакрусу, т.е. найти те ударные слоги в начале строк, которые попадают на икт.

В начале рифмующихся строк совпадающие места ударных слогов (1-го или 2-го) приходятся на икт: 
- первого слога в строке 1 с первым слогом в строке 2 (при $1 \leq|\mathrm{V}|$ между первым и вторым слогами в каждой строке;

- второго слога в строке 1 и в строке 2, а места первого слога в строке 1 и в строке 2 не совпадают (при $|\mathrm{V}|=0$ или $|\mathrm{V}|=1$ между первым и вторым слогами);

- второго слога и в первого сверхсхемного слога в строке 1 со вторым слогом и первым сверхсхемным слогом в строке 2 (при $|\mathrm{V}|=0$ между первым и вторым слогами)

- первого слога в строке 1 со вторым слогом в строке 2 (при $|\mathrm{V}|=0$ или $|\mathrm{V}|=1$ между первым сверхсхемным и вторым слогами);

- первого слога в строке 2 (при $1 \leq|\mathrm{V}|$ между первым и вторым слогами) с пропуском икта на том же месте в строке 1.

Если для всех строк текста единая метрическая анакруса $r(1)$ не находится, то принимается силлабическая метрика текста, и дополнительно определяется положение возможной цезуры в соответствии с формулами (14).

Если выявленная анакруса устанавливается для всех строк текста, то силлабическая метрика отвергается, и производится, так же, как и для Шага 1.2., разрешение альтернативы между силлабо-тоническим стихом и строчным логаэдом.

Во второй очереди разрешение альтернативы между силлабо-тоническим стихом и строчным логаэдом путем поиска для рифмующихся строк одинакового объема совпадающих мест ударных слогов от первого икта, найденного по вышеприведенной процедуре, до исходно известного последнего. Если на основе иктов, выявленных по совпадению мест ударных слогов для всех строк текста, окажется, что междуиктовые интервалы одинаковы $|\mathrm{V}|$ const или (1+2|V|const ) (при пропуске ударного слога на икте), то принимается силлабо-тоническая метрика текста.

Если же при совпадении иктов междуиктовые интервалы различаются иным образом, но одинаково для всех соотносимых строк, то в качестве метрики текста принимается логаэд, в котором пропуск ударного слога на икте недопустим. В соизмеримых логаэдических строках для подцепочек от начала стиха до (j)-го ударного слога, $\mathrm{j}=1,2, \ldots$ (z$1)$, в строке с z ударных слогов $F C(\mathrm{j})=\left(\prod \mathrm{j}=1,2, . .(\mathrm{z}-1) \mathrm{cq}(\mathrm{j}-1) \mathrm{C}(\mathrm{j}-1) \operatorname{ch}(\mathrm{j}-1)\right) \mathrm{cq}(\mathrm{j})$, со слоговым объемом $|\mathrm{FC}(\mathrm{j})|=\left(\mathrm{j}-1+\mathrm{q}(\mathrm{j})+\sum \mathrm{i}=1,2, \ldots(\mathrm{z}-1)(\mathrm{q}(\mathrm{j}-1)+\mathrm{h}(\mathrm{j}-1))\right)$, и до (m)-го ударного слога, $\mathrm{m}=1,2, \ldots(\mathrm{n}-1)$, в строке с $\mathrm{n}$ ударных слогов $\mathrm{FG}(\mathrm{m})=\left(\prod \mathrm{m}=1,2, . .(\mathrm{n}-1) \mathrm{gq}(\mathrm{m}-1) \mathrm{G}(\mathrm{m}-1) \mathrm{gh}(\mathrm{m}-\right.$ 1))gq(m), со слоговым объемом $|\mathrm{FG}(\mathrm{m})|=\left(\mathrm{m}-1+\mathrm{q}(\mathrm{m})+\sum \mathrm{m}=1,2, \ldots(\mathrm{n}-1)(\mathrm{q}(\mathrm{m}-1)+\mathrm{h}(\mathrm{m}-1))\right)$, при совпадении их объемов $|\mathrm{FC}(\mathrm{j}+1)|=|\mathrm{FG}(\mathrm{m}+1)|$ и соответственно $\left(\mathrm{j}-1+\mathrm{q}(\mathrm{j})+\sum \mathrm{i}=1,2, \ldots(\mathrm{z}-1)(\mathrm{q}(\mathrm{j}-\right.$ $1)+\mathrm{h}(\mathrm{j}-1)))=\left(\mathrm{m}-1+\mathrm{q}(\mathrm{m})+\sum \mathrm{m}=1,2, \ldots(\mathrm{n}-1)(\mathrm{q}(\mathrm{m}-1)+\mathrm{h}(\mathrm{m}-1))\right) \quad$ ударные $\quad$ слоги $\quad \mathrm{j} \quad$ и $\mathrm{m}$ соответствующих строк попадают на икт.

Для рифмованных строк с неравными слоговыми объемами основ $|\mathrm{W}|$ и с равным числом ударных слогов $\mathrm{z}$ по Шагу 1.3. альтернатива разрешается определением вида тонического стиха с заданной вариативностью междуиктовых интервалов $\mathrm{v}$, без сверхсхемных ударений и без пропуска ударений на иктах (см. ниже раздел 4.2.).

Для рифмованных строк с неравными слоговыми объемами основ $|\mathrm{W}|$ и с неравным числом ударных слогов $\mathrm{z}$ по Шагу 1.4. осуществляется разрешение альтернативы между вольным силлабо-тоническим стихом, тоническим стихом (с заданной вариативностью междуиктовых интервалов $\mathrm{v}, \mathrm{c}$ наличием сверхсхемных ударений и пропусков ударений на иктах) и раешным стихом.

Для начала следует выявить по приведенной выше процедуре метрическую анакрусу, т.е. найти те ударные слоги в начале строк, которые попадают на икт. Если для всех строк текста находится единая метрическая анакруса $\mathrm{r}(1)$, то принимается вариант вольной силлабо-тонической метрики текста.

Если для всех строк текста единой метрической анакрусы не находится, то осуществляется разрешение альтернативы между тоническим стихом (с заданной вариативностью междуиктовых интервалов $\mathrm{v}$, с наличием сверхсхемных ударений и пропусков ударений на иктах) и раешным стихом. 
Для не рифмованного текста с равными слоговыми объемами основ строк $|\mathrm{W}|=$ const согласно Шагу 2.1. нет альтернативы белому силлабо-тоническому стиху.

Для не рифмованного текста с неравными слоговыми объемами основ строк $|\mathrm{W}| \neq \mathrm{const}$ согласно Шагу 2.2. разрешение альтернативы между вольным белым силлабо-тонический стихом и свободным стихом достигается выявлением по приведенной выше процедуре метрической анакрусы. Если для всех строк текста находится единая метрическая анакруса $\mathrm{r}_{(1)}$, то принимается вариант вольного белого силлабо-тонической стиха. В противном случае следует принять вариант свободного стиха.

\section{4. Поиск ударных слогов на икте по метрическому репертуару стиха}

Для начала в соотносимых и соизмеримых строках необходимо вычислить такой показатель, как верхний целочисленный предел среднего значения объема метрометрического периода (ударный слог с междуакцентным интервалом) или регулятор метра

(25) $\left\lceil\tilde{\mathrm{U}}_{\mathrm{C}}\right\rceil=\left\lceil\mid \mathrm{W}_{\mathrm{C}} / /(\mathrm{z}-1)\right\rceil$.

Далее можно найти фонетические слова в строке $\left.\mathrm{P}_{\mathrm{Cj}}=\mathrm{c}^{\mathrm{q(j)}} \mathrm{C}_{\mathrm{j}} \mathrm{c}^{\mathrm{h}(\mathrm{j})}: 1\right)$ с общим объемом слова $\left.\left|\mathrm{P}_{\mathrm{Cj}}\right|=\left(\mathrm{q}_{(\mathrm{j})}+\mathrm{h}_{(\mathrm{j})}+1\right) \geq\left|\tilde{U}_{\mathrm{C}}\right| ; 2\right)$ с объемом предударной части $\left.\mathrm{q}_{(\mathrm{j})} \geq\left|\mathrm{V}_{\mathrm{C}}\right|_{\max } ; 3\right)$ с объемом заударной части $\mathrm{h}_{(\mathrm{j})} \geq\left|\mathrm{V}_{\mathrm{C}}\right|_{\max }$. В соответствии с ОПРЕДЕЛЕНИЕМ метра: ударение слова с объемом 1) падает на икт А; в предударной части слов с объемом 2) содержится пропуск ударного слога на икте; в заударной части слов с объемом 3) содержится пропуск ударного слога на икте.

Можно предположить, что процедура выявления ударений в строке, приходящихся на икты, существенным образом зависит от вида системы стихосложения, которому принадлежит метр исследуемой строки. Поэтому следует рассмотреть в слоговом представлении стиха соотношение слоговых объемов сегментов, соответствующих формулам (17) $\left|\mathrm{P}_{\mathrm{Cj}}\right|=\left(\mathrm{q}_{(\mathrm{j})}+\mathrm{h}_{(\mathrm{j})}+1\right), \quad(19) \quad\left|\mathrm{F}_{\mathrm{C}}\right|=\left(\mathrm{z}+\sum_{\mathrm{j}=1,2, \ldots \mathrm{z}}\left(\mathrm{q}_{(\mathrm{j})}+\mathrm{h}_{(\mathrm{j})}\right)\right), \quad$ (22) $\quad\left|\mathrm{V}_{\mathrm{Cj}}\right|=\left(\mathrm{h}_{(\mathrm{j})}+\mathrm{q}_{(\mathrm{j}+1)}\right)$, $\left|\mathrm{V}_{\mathrm{C}}\right|_{\min } \leq\left|\mathrm{V}_{\mathrm{Cj}}\right| \leq\left|\mathrm{V}_{\mathrm{C}}\right|_{\max }, \quad(23) \quad\left|\mathrm{W}_{\mathrm{C}}\right|=\left(\mathrm{z}-1+\sum_{\mathrm{j}=1,2, \ldots(\mathrm{z}-1)}\left(\mathrm{h}_{(\mathrm{j})}+\mathrm{q}_{(\mathrm{j}+1)}\right)\right) \quad$ и $\quad$ (25) $\quad\left[\tilde{\mathrm{U}}_{\mathrm{C}} \mid=\left\lceil\left|\mathrm{W}_{\mathrm{C}}\right| /(\mathrm{z}-1)\right\rceil \quad\right.$ для каждого вида метра в репертуаре систем стихосложения.

Будем иметь ввиду, что для $\mathrm{P}_{\mathrm{Cj}}=\mathrm{c}^{\mathrm{q}(\mathrm{j})} \mathrm{C}_{\mathrm{j}} \mathrm{c}^{\mathrm{h}(\mathrm{j})}$ со слоговым объемом $\left|\mathrm{P}_{\mathrm{Cj}}\right|=\left(\mathrm{q}_{(\mathrm{j} 0}+\mathrm{h}_{(\mathrm{j})}+1\right) \geq\left\lceil\tilde{\mathrm{U}}_{\mathrm{C}}\right]$ имеет место ударение на икте. При $\mathrm{q}_{(\mathrm{j})} \geq\left|\mathrm{V}_{\mathrm{C}}\right|_{\max }$ или $\mathrm{h}_{(\mathrm{j})} \geq\left|\mathrm{V}_{\mathrm{C}}\right|_{\max }$ имеет место пропуск ударения в междуиктовом интервале в предударной части и заударной части слова соответственно.

\section{1. Силлабо-тонические метры}

Метры с равными стопами $\left|\mathrm{U}_{\mathrm{Ai}}\right|=\left|\mathrm{U}_{\mathrm{A}}\right|_{\max }=\left|\mathrm{U}_{\mathrm{A}}\right|_{\min }=\left(1+\mathrm{s}_{(\mathrm{i})}+\mathrm{r}_{(\mathrm{i}+1)}\right)=$ const: строгие k-стопные метры при $\mathrm{k}=\mathrm{const}$ разностопные (вольные) метры при $\mathrm{k} \neq \mathrm{const}$; $\left|\mathrm{V}_{\mathrm{Ai}}\right|=\left|\mathrm{V}_{\mathrm{A}}\right|_{\max }=\left|\mathrm{V}_{\mathrm{A}}\right|_{\min }=\left(\mathrm{s}_{(\mathrm{i})}+\mathrm{r}_{(\mathrm{i}+1)}\right)=$ const; и вариативность объема междуиктовых интервалов $\mathrm{v}_{\mathrm{A}}=\left|\mathrm{V}_{\mathrm{A}}\right|_{\min }-\left|\mathrm{V}_{\mathrm{A}}\right|_{\max }=\left|\mathrm{U}_{\mathrm{A}}\right|_{\max }-\left|\mathrm{U}_{\mathrm{Ai}}\right|_{\min }=0 ; \mathrm{s}(\mathrm{k})=0,1,2$.

Метры с двусложной стопой: ямб $\mathrm{r}(1)=1$, хорей $\mathrm{r}(1)=0$.

Объемные показатели метрической схемы: $\mathrm{k} ;\left|\mathrm{U}_{\mathrm{Ai}}\right|=2 ;\left|\mathrm{V}_{\mathrm{Ai}}\right|=1 ;\left|\mathrm{W}_{\mathrm{A}}\right|=2(\mathrm{k}-1) ;\left[\tilde{\mathrm{U}}_{\mathrm{A}} \mid=2\right.$.

Объемные показатели при пропуске ударного слога на g-м икте: $\mathrm{z}=(\mathrm{k}-1)$; $\left|\mathrm{U}_{\mathrm{Ci}}\right|=2$, $\mathrm{i}=1,2, \ldots(\mathrm{k}-1)$ и $\mathrm{i} \neq(\mathrm{g}-1),\left|\mathrm{U}_{\mathrm{C}(\mathrm{g}-1)}\right|=4,\left|\mathrm{U}_{\mathrm{C}}\right|_{\max }=4,\left|\mathrm{U}_{\mathrm{C}}\right|_{\min }=2 ;\left|\mathrm{V}_{\mathrm{C}}\right|_{\max }=3,\left|\mathrm{~V}_{\mathrm{C}}\right|_{\min }=1, \mathrm{v}_{\mathrm{C}}=2 ;\left|\mathrm{W}_{\mathrm{C}}\right|=2(\mathrm{k}-1)$; $\left\lceil\tilde{\mathrm{U}}_{\mathrm{C}}\right\rceil=\lceil 2(\mathrm{k}-1) /(\mathrm{k}-2)\rceil=3$ при $\mathrm{k} \geq 4\left(\left\lceil\tilde{\mathrm{U}}_{\mathrm{C}}\right\rceil=4\right.$ при $\left.\mathrm{k}=3\right)$.

Для слова $\mathrm{P}_{\mathrm{Cj}}$ со слоговым объемом $\left|\mathrm{P}_{\mathrm{Ci}}\right|=\left(\mathrm{q}_{(\mathrm{i})}+\mathrm{h}_{(\mathrm{i})}+1\right) \geq 3$ имеет место ударение на икте. При $\mathrm{q}_{(\mathrm{i})} \geq 3$ или $\mathrm{h}_{(\mathrm{i})} \geq 3$ имеет место пропуск ударения на икте в предударной части или заударной части соответственно.

Объемные показатели при сверхсхемном ударении на g-м междуиктовом интервале: $\mathrm{Z}=(\mathrm{k}+1) ;\left|\mathrm{U}_{\mathrm{Ci}}\right|=2, \mathrm{i}=1,2, \ldots(\mathrm{k}+1)$ и $\mathrm{i} \neq \mathrm{g},(\mathrm{g}+1),\left|\mathrm{U}_{\mathrm{Cg}}\right|=1,\left|\mathrm{U}_{\mathrm{C}(\mathrm{g}+1)}\right|=1,\left|\mathrm{U}_{\mathrm{C}}\right|_{\max }=2,\left|\mathrm{U}_{\mathrm{C}}\right|_{\min }=1 ;\left|\mathrm{V}_{\mathrm{A}}\right|_{\max }=1$, $\left.\left|\mathrm{V}_{\mathrm{A}}\right|_{\min }=0, \mathrm{v}_{\mathrm{C}}=1 ;\left|\mathrm{W}_{\mathrm{C}}\right|=2(\mathrm{k}-1) ; \mid \tilde{\mathrm{U}}_{\mathrm{C}}\right]=\lceil 2(\mathrm{k}-1) / \mathrm{k} \mid=2$ и $\mathrm{k} \geq 3$.

Для слова $\mathrm{P}_{\mathrm{Cj}}$ со слоговым объемом $\left|\mathrm{P}_{\mathrm{Cj}}\right|=\left(\mathrm{q}_{(\mathrm{i})}+\mathrm{h}_{(\mathrm{i})}+1\right) \geq 2$ имеет место ударение на икте. 
Объемные показатели при одновременном пропуске ударения на икте и сверхсхемном ударении: $\mathrm{z}=\mathrm{k} ;\left|\mathrm{U}_{\mathrm{C}}\right|_{\max }=4,\left|\mathrm{U}_{\mathrm{C}}\right|_{\min }=1 ;\left|\mathrm{V}_{\mathrm{C}}\right|_{\max }=3,\left|\mathrm{~V}_{\mathrm{C}}\right|_{\min }=0, \mathrm{v}_{\mathrm{C}}=3 ;\left|\mathrm{W}_{\mathrm{C}}\right|=2(\mathrm{k}-1)$; $\left.\mid \tilde{U}_{\mathrm{C}}\right\rceil=\lceil 2(\mathrm{k}-1) /(\mathrm{k}-$ 1) $]=2$ и $\mathrm{k} \geq 2$.

Для слова $\mathrm{P}_{\mathrm{Ci}}$ со слоговым объемом $\left|\mathrm{P}_{\mathrm{Ci}}\right|=\left(\mathrm{q}_{(\mathrm{i})}+\mathrm{h}_{(\mathrm{i})}+1\right) \geq 2$ имеет место ударение на икте. При $\mathrm{q}_{(\mathrm{i})} \geq 3$ или $\mathrm{h}_{(\mathrm{i})} \geq 3$ имеет место пропуск ударения на икте в предударной части или заударной части соответственно.

Метры с трехсложной стопой: дактиль $\mathrm{r}(1)=0$, амфибрахий $\mathrm{r}(1)=1$, анапест $\mathrm{r}(1)=2$.

Объемные показатели для метрической схемы: $\mathrm{k} ;\left|\mathrm{U}_{\mathrm{Ai}}\right|=3 ;\left|\mathrm{V}_{\mathrm{Ai}}\right|=2 ;\left|\mathrm{W}_{\mathrm{A}}\right|=3(\mathrm{k}-1) ;\left[\tilde{\mathrm{U}}_{\mathrm{A}} \mid=3\right.$.

Объемные показатели при пропуске ударного слога на g-м икте: $\mathrm{z}=(\mathrm{k}-1)$; $\left|\mathrm{U}_{\mathrm{Ci}}\right|=3$, $\mathrm{i}=1,2, \ldots(\mathrm{k}-1)$ и $\mathrm{i} \neq(\mathrm{g}-1),\left|\mathrm{U}_{\mathrm{C}(\mathrm{g}-1)}\right|=6,\left|\mathrm{U}_{\mathrm{C}}\right|_{\max }=6,\left|\mathrm{U}_{\mathrm{C}}\right|_{\min }=3 ;\left|\mathrm{V}_{\mathrm{C}}\right|_{\max }=5,\left|\mathrm{~V}_{\mathrm{C}}\right|_{\min }=2, \mathrm{v}_{\mathrm{C}}=3 ;|\mathrm{W}|=3(\mathrm{k}-1)$; $\left\lceil\tilde{U}_{\mathrm{C}}\right\rceil=\lceil 3(\mathrm{k}-1) /(\mathrm{k}-2)\rceil=4$ при $\mathrm{k} \geq 5\left(\left\lceil\tilde{\mathrm{U}}_{\mathrm{C}}\right\rceil=6\right.$ при $\mathrm{k}=3,\left(\left\lceil\tilde{\mathrm{U}}_{\mathrm{C}}\right\rceil=5\right.$ при $\left.\mathrm{k}=4\right)$.

Для слова $\mathrm{P}_{\mathrm{Ci}}$ со слоговым объемом $\left|\mathrm{P}_{\mathrm{Ci}}\right|=\left(\mathrm{q}_{(\mathrm{i})}+\mathrm{h}_{(\mathrm{i})}+1\right) \geq 4$ имеет место ударение на икте. При $\mathrm{q}_{(\mathrm{i})} \geq 5$ или $\mathrm{h}_{(\mathrm{i})} \geq 5$ имеет место пропуск ударения на икте в предударной части или заударной части соответственно.

Объемные показатели при сверхсхемном ударении на g-м междуиктовом интервале: $\mathrm{z}=(\mathrm{k}+1) ;\left|\mathrm{U}_{\mathrm{Ci}}\right|=3, \mathrm{i}=1,2, \ldots(\mathrm{k}+1)$ и $\mathrm{i} \neq \mathrm{g},(\mathrm{g}+1),\left(\left|\mathrm{U}_{\mathrm{Cg}}\right|=2\right.$ и $\left.\left|\mathrm{U}_{\mathrm{C}(\mathrm{g}+1)}\right|=1\right)$ или $\left(\left|\mathrm{U}_{\mathrm{Cg}}\right|=1\right.$ и $\left.\left|\mathrm{U}_{\mathrm{C}(\mathrm{g}+1)}\right|=2\right)$, $\left|\mathrm{U}_{\mathrm{C}}\right|_{\max }=3,\left|\mathrm{U}_{\mathrm{C}}\right|_{\min }=1 ;\left|\mathrm{V}_{\mathrm{C}}\right|_{\max }=2,\left|\mathrm{~V}_{\mathrm{C}}\right|_{\min }=0, \mathrm{v}_{\mathrm{C}}=2 ; ;|\mathrm{W}|=3(\mathrm{k}-1) ; \quad\left[\tilde{\mathrm{U}}_{\mathrm{C}} \mid=\lceil 3(\mathrm{k}-1) / \mathrm{k}\rceil=3\right.$ при $\mathrm{k} \geq 4$ ( $\left[\tilde{\mathrm{U}}_{\mathrm{C}}=2\right.$ при $\mathrm{k}=3$ ).

Для слова $\mathrm{P}_{\mathrm{Ci}}$ со слоговым объемом $\left|\mathrm{P}_{\mathrm{Ci}}\right|=\left(\mathrm{q}_{(\mathrm{i})}+\mathrm{h}_{(\mathrm{i})}+1\right) \geq 3$ имеет место ударение на икте.

Объемные показатели при одновременном пропуске ударения на икте и сверхсхемном ударении: $\mathrm{z}=\mathrm{k} ;\left|\mathrm{U}_{\mathrm{C}}\right|_{\max }=6,\left|\mathrm{U}_{\mathrm{C}}\right|_{\min }=1 ;\left|\mathrm{V}_{\mathrm{C}}\right|_{\max }=5,\left|\mathrm{~V}_{\mathrm{C}}\right|_{\min }=0, \mathrm{v}_{\mathrm{C}}=5 ;\left|\mathrm{W}_{\mathrm{C}}\right|=3(\mathrm{k}-1) ;\left[\tilde{\mathrm{U}}_{\mathrm{C}} \mid=[3(\mathrm{k}-1) /(\mathrm{k}-\right.$ 1) $]=3$ и $\mathrm{k} \geq 3$.

Для слова $\mathrm{P}_{\mathrm{Ci}}$ со слоговым объемом $\left|\mathrm{P}_{\mathrm{Ci}}\right|=\left(\mathrm{q}_{(\mathrm{i})}+\mathrm{h}_{(\mathrm{i})}+1\right) \geq 3$ имеет место ударение на икте. При $\mathrm{q}_{(\mathrm{i})} \geq 5$ или $\mathrm{h}_{(\mathrm{i})} \geq 5$ имеет место пропуск ударения на икте в предударной части или заударной части соответственно.

Метры с четырехсложной стопой - пэоны: пэон-I при $\mathrm{r}(1)=0$, пэон-II при $\mathrm{r}(1)=1$, пэон-III при $\mathrm{r}(1)=2$, пэон-IV при $\mathrm{r}(1)=3$.

Объемные показатели для метрической схемы: $\mathrm{k} ;\left|\mathrm{U}_{\mathrm{Ai}}\right|=4 ;\left|\mathrm{V}_{\mathrm{Ai}}\right|=3 ;\left|\mathrm{W}_{\mathrm{A}}\right|=4(\mathrm{k}-1) ;\left[\tilde{\mathrm{U}}_{\mathrm{A}}\right]=4$.

Пропуск ударного слога на g-м икте с последующим $\left|\mathrm{V}_{\mathrm{C}(\mathrm{g}-1)}\right|=7$ исключителен с учетом ограничения $|\mathrm{V}|_{\max } \leq 8$.

Объемные показатели при сверхсхемном ударении на g-м междуиктовом интервале: $\mathrm{z}=(\mathrm{k}+1) ;\left|\mathrm{U}_{\mathrm{Ci}}\right|=4, \mathrm{i}=1,2, \ldots(\mathrm{k}+1)$ и $\mathrm{i} \neq \mathrm{g},(\mathrm{g}+1),\left(\left|\mathrm{U}_{\mathrm{Cg}}\right|=3\right.$ и $\left.\left|\mathrm{U}_{\mathrm{C}(\mathrm{g}+1)}\right|=1\right)$ или $\left(\left|\mathrm{U}_{\mathrm{Cg}}\right|=2\right.$ и $\left.\left|\mathrm{U}_{\mathrm{C}(\mathrm{g}+1)}\right|=2\right)$ или $\left(\left|\mathrm{U}_{\mathrm{Cg}}\right|=2\right.$ и $\left.\left|\mathrm{U}_{\mathrm{C}(\mathrm{g}+1)}\right|=2\right),\left|\mathrm{U}_{\mathrm{C}}\right|_{\max }=4,\left|\mathrm{U}_{\mathrm{C}}\right|_{\min }=1 ;\left|\mathrm{V}_{\mathrm{C}}\right|_{\max }=3,\left|\mathrm{~V}_{\mathrm{C}}\right|_{\min }=0, \mathrm{v}_{\mathrm{C}}=3 ; ;|\mathrm{W}|=4(\mathrm{k}-1)$; $\left\lceil\tilde{\mathrm{U}}_{\mathrm{C}}\right\rceil=\lceil 4(\mathrm{k}-1) / \mathrm{k}\rceil=4$ при $\mathrm{k} \geq 5\left(\left\lceil\tilde{\mathrm{U}}_{\mathrm{C}}\right\rceil=2\right.$ при $\mathrm{k}=2$, $\left\lceil\tilde{\mathrm{U}}_{\mathrm{C}}\right\rceil=3$ при $\mathrm{k}=3$ и $\left.\mathrm{k}=4\right)$.

Для слова $\mathrm{P}_{\mathrm{Cj}}$ со слоговым объемом $\left|\mathrm{P}_{\mathrm{Ci}}\right|=\left(\mathrm{q}_{(\mathrm{i})}+\mathrm{h}_{(\mathrm{i})}+1\right) \geq 4$ имеет место ударение на икте.

Метры с пятисложной стопой - пентоны: пентон-I при $\mathrm{r}(1)=0$, пентон-II при $\mathrm{r}(1)=1$, пентон-III при $\mathrm{r}(1)=2$, пентон-IV при $\mathrm{r}(1)=3$, пентон-V при $\mathrm{r}(1)=4$.

Объемные показатели для метрической схемы: $\mathrm{k} ;\left|\mathrm{U}_{\mathrm{Ai}}\right|=5 ;\left|\mathrm{V}_{\mathrm{Ai}}\right|=4 ;\left|\mathrm{W}_{\mathrm{A}}\right|=5(\mathrm{k}-1) ;\left[\tilde{\mathrm{U}}_{\mathrm{A}} \mid=5\right.$.

Пропуск ударного слога на g-м икте с последующим $\left|\mathrm{V}_{\mathrm{g}}\right|=9$ исключен с учетом ограничения $\left|\mathrm{V}_{\mathrm{i}}\right|_{\max } \leq 8$.

Объемные показатели при сверхсхемном ударении на g-м междуиктовом интервале: $\mathrm{z}=(\mathrm{k}+1) ;\left|\mathrm{U}_{\mathrm{Ci}}\right|=5, \mathrm{i}=1,2, \ldots(\mathrm{k}+1)$ и $\mathrm{i} \neq \mathrm{g},(\mathrm{g}+1),\left(\left|\mathrm{U}_{\mathrm{Cg}}\right|=4\right.$ и $\left.\left|\mathrm{U}_{\mathrm{C}(\mathrm{g}+1)}\right|=1\right)$ или $\left(\left|\mathrm{U}_{\mathrm{Cg}}\right|=3\right.$ и $\left.\left|\mathrm{U}_{\mathrm{C}(\mathrm{g}+1)}\right|=2\right)$ или $\left(\left|\mathrm{U}_{\mathrm{Cg}}\right|=2\right.$ и $\left.\left|\mathrm{U}_{\mathrm{C}(\mathrm{g}+1)}\right|=3\right)$ или $\left(\left|\mathrm{U}_{\mathrm{Cg}}\right|=1\right.$ и $\left.\left|\mathrm{U}_{\mathrm{C}(\mathrm{g}+1)}\right|=4\right),\left|\mathrm{U}_{\mathrm{C}}\right|_{\max }=5,\left|\mathrm{U}_{\mathrm{C}}\right|_{\min }=1 ;\left|\mathrm{V}_{\mathrm{C}}\right|_{\max }=4,\left|\mathrm{~V}_{\mathrm{C}}\right|_{\min }=0$, $\mathrm{v}_{\mathrm{C}}=3 ; ;|\mathrm{W}|=5(\mathrm{k}-1) ;\left\lceil\tilde{\mathrm{U}}_{\mathrm{C}}\right\rceil=\lceil 5(\mathrm{k}-1) / \mathrm{k}\rceil=5$ при $\mathrm{k} \geq 6\left(\left\lceil\tilde{\mathrm{U}}_{\mathrm{C}}\right\rceil=3\right.$ при $\mathrm{k}=2,\left\lceil\tilde{\mathrm{U}}_{\mathrm{C}}\right\rceil=4$ при $\left.\mathrm{k}=3,4,5\right)$.

Для слова $\mathrm{P}_{\mathrm{Ci}}$ со слоговым объемом $\left|\mathrm{P}_{\mathrm{C}}\right|=\left(\mathrm{q}_{(\mathrm{i})}+\mathrm{h}_{(\mathrm{i})}+1\right) \geq 5$ имеет место ударение на икте. При $\mathrm{q}_{(\mathrm{i})} \geq 5$ или $\mathrm{h}_{(\mathrm{i})} \geq 5$ имеет место пропуск ударения на икте в предударной части или заударной части соответственно.

Метр с шестисложной стопой - гексон.

Объемные показатели для метрической схемы: $\mathrm{k} ;\left|\mathrm{U}_{\mathrm{Ai}}\right|=6 ;\left|\mathrm{V}_{\mathrm{Ai}}\right|=5 ;\left|\mathrm{W}_{\mathrm{A}}\right|=6(\mathrm{k}-1) ;\left[\tilde{\mathrm{U}}_{\mathrm{A}}\right]=6$.

Регулятивы: k; $\left|\mathrm{U}_{\mathrm{i}}\right|=6 ;\left|\mathrm{V}_{\mathrm{i}}\right|=5 ;|\mathrm{W}|=(6 \mathrm{k}-5) ; \mathrm{s}(\mathrm{k})=0,1,2$. 
Пропуск ударного слога на g-м икте икте с последующим $\left|\mathrm{V}_{\mathrm{g}}\right|=11$ исключен с учетом ограничения $\left|\mathrm{V}_{\mathrm{j}}\right|_{\max } \leq 8$.

Вариации сверхсхемных акцентов чрезвычайно редки.

\section{2. Тонические метры (включая метры с переменным объемом междиктовых интервалов)}

Метры со стопами разного объема $\left|\mathrm{U}_{\mathrm{Ai}}\right| \neq$ const: строгие метры при $\mathrm{k}=$ const; вольные метры при $\mathrm{k} \neq$ const. Для каждого вида метров $\left|\mathrm{V}_{\mathrm{Ai}}\right| \neq c o n s t$ и вариативность объема междуиктовых интервалов $\mathrm{v}_{\mathrm{A}} \neq 0$. Возможны такие отклонения от метра, как пропуски акцентов на иктах и сверхсхемные ударения на слабых местах при $\left|\mathrm{V}_{\mathrm{Ai}}\right| \neq 0$.

Дольники - метры с вариативностью объема междуиктовых интервалов $\mathrm{v}_{\mathrm{A}}=1$, a для каждого вида метров $\mathrm{r}_{\mathrm{A}}(1) \neq$ const, $\left|\mathrm{S}_{\mathrm{Ai}}\right| \neq$ const, $\left|\mathrm{V}_{\mathrm{Ai}}\right| \neq$ const, $\left|\mathrm{W}_{\mathrm{A}}\right| \neq$ const, $\mathrm{s}_{\mathrm{A}}(\mathrm{k})=0,1,2$.

Дольник-1.

Объемные показатели метрической схемы: $\mathrm{k} \geq 2 ; \mathrm{r}_{\mathrm{A}}(1) \leq 2 ; 2 \leq\left|\mathrm{U}_{\mathrm{Ai}}\right| \leq 3 ; 1 \leq\left|\mathrm{V}_{\mathrm{Ai}}\right| \leq 2 ; 2$ (k$1) \leq\left|\mathrm{W}_{\mathrm{A}}\right|=\sum_{\mathrm{i}=1,2, \ldots(\mathrm{k}-1)}\left|\mathrm{U}_{\mathrm{Ai}}\right| \leq 3(\mathrm{k}-1) ; 2 \leq\left|\tilde{\mathrm{U}}_{\mathrm{A}}\right| \leq 3$.

Объемные показатели при пропуске ударного слога на g-м икте: $\mathrm{z}=(\mathrm{k}-1) ; 2 \leq\left|\mathrm{U}_{\mathrm{Ci}}\right| \leq 3$, $\mathrm{i}=1,2, \ldots(\mathrm{k}-1)$ и $\mathrm{i} \neq(\mathrm{g}-1), 4 \leq\left|\mathrm{U}_{\mathrm{C}(\mathrm{g}-1)}\right| \leq 6 ; \quad\left|\mathrm{U}_{\mathrm{C}}\right|_{\max }=6, \quad\left|\mathrm{U}_{\mathrm{C}}\right|_{\min }=2 ; \quad\left|\mathrm{V}_{\mathrm{C}}\right|_{\max }=5, \quad\left|\mathrm{~V}_{\mathrm{C}}\right|_{\min }=1, \mathrm{v}_{\mathrm{C}}=4 ; \quad 2(\mathrm{k}-$ $1) \leq\left|\mathrm{W}_{\mathrm{C}}\right| \leq 3(\mathrm{k}-1), 3 \leq\left|\tilde{\mathrm{U}}_{\mathrm{C}}\right|=\left|\mathrm{W}_{\mathrm{C}}\right| /(\kappa-2) \leq 4$.

Для слова $\mathrm{P}_{\mathrm{Ci}}$ со слоговым объемом $\left|\mathrm{P}_{\mathrm{Cj}}\right|=\left(\mathrm{q}_{(\mathrm{i})}+\mathrm{h}_{(\mathrm{i})}+1\right) \geq 4$ имеет место ударение на икте. При $\mathrm{q}_{(\mathrm{i})} \geq 5$ или $\mathrm{h}_{(\mathrm{i})} \geq 5$ имеет место пропуск ударения на икте в предударной части или заударной части соответственно.

Объемные показатели при сверхсхемном ударении на g-м междуиктовом интервале: $\mathrm{z}=(\mathrm{k}+1) ; \quad 2 \leq\left|\mathrm{U}_{\mathrm{Ci}}\right| \leq 3, \quad \mathrm{i}=1,2, \ldots(\mathrm{k}+1) \quad$ и $\quad \mathrm{i} \neq \mathrm{g},(\mathrm{g}+1), \quad 2 \leq\left(\left|\mathrm{U}_{\mathrm{Cg}}\right|+\left.\mathrm{U}_{\mathrm{C}(\mathrm{g}+1)}|\leq 3, \quad| \mathrm{U}_{\mathrm{C}}\right|_{\max }=3, \quad\left|\mathrm{U}_{\mathrm{C}}\right|_{\min }=1 ;\right.$ $\left|\mathrm{V}_{\mathrm{C}}\right|_{\max }=2,\left|\mathrm{~V}_{\mathrm{C}}\right|_{\min }=0, \mathrm{v}_{\mathrm{C}}=2 ; 2(\mathrm{k}-1) \leq\left|\mathrm{W}_{\mathrm{C}}\right| \leq 3(\mathrm{k}-1), 2(\mathrm{k}-1) / \mathrm{k} \leq\left[\tilde{\mathrm{U}}_{\mathrm{C}} \mid \leq\lceil 3(\mathrm{k}-1) / \mathrm{k}\rceil, 2 \leq\left\lceil\tilde{\mathrm{U}}_{\mathrm{C}} \mid \leq 3\right.\right.$.

Для слова $\mathrm{P}_{\mathrm{Cj}}$ со слоговым объемом $\left|\mathrm{P}_{\mathrm{Ci}}\right|=\left(\mathrm{q}_{(\mathrm{i})}+\mathrm{h}_{(\mathrm{i})}+1\right) \geq 3$ имеет место ударение на икте.

Объемные показатели при одновременном пропуске ударения на икте и сверхсхемном ударении: $\mathrm{z}=\mathrm{k} ; \quad\left|\mathrm{U}_{\mathrm{C}}\right|_{\max }=6, \quad\left|\mathrm{U}_{\mathrm{C}}\right|_{\min }=1 ; \quad\left|\mathrm{V}_{\mathrm{C}}\right|_{\max }=5, \quad\left|\mathrm{~V}_{\mathrm{C}}\right|_{\min }=0, \quad \mathrm{v}_{\mathrm{C}}=5 ; \quad 2(\mathrm{k}-1) \leq\left|\mathrm{W}_{\mathrm{C}}\right| \leq 3(\mathrm{k}-1)$; $2 \leq\left\lceil\tilde{U}_{\mathrm{C}}|=| \mathrm{W}_{\mathrm{C}} \mid /(\mathrm{k}-1) \leq 3\right.$ и $\mathrm{k} \geq 2$.

Для слова $\mathrm{P}_{\mathrm{Cj}}$ со слоговым объемом $\left|\mathrm{P}_{\mathrm{Ci}}\right|=\left(\mathrm{q}_{(\mathrm{i})}+\mathrm{h}_{(\mathrm{i})}+1\right) \geq 3$ имеет место ударение на икте. При $\mathrm{q}_{(\mathrm{i})} \geq 5$ или $\mathrm{h}_{(\mathrm{i})} \geq 5$ имеет место пропуск ударения на икте в предударной части или заударной части соответственно.

Дольник-2.

Объемные показатели метрической схемы: $\mathrm{k} \geq 2 ; \mathrm{r}_{\mathrm{A}}(1) \leq 1 ; \quad 1 \leq\left|\mathrm{U}_{\mathrm{Ai}}\right| \leq 2 ; \quad 0 \leq\left|\mathrm{V}_{\mathrm{Ai}}\right| \leq 1 ; \quad$ (k$1) \leq\left|\mathrm{W}_{\mathrm{A}}\right|=\sum_{\mathrm{i}=1,2, \ldots(\mathrm{k}-1)}\left|\mathrm{U}_{\mathrm{Ai}}\right| \leq 2(\mathrm{k}-1) ; 1 \leq\left|\tilde{\mathrm{U}}_{\mathrm{A}}\right| \leq 2$.

Объемные показатели при пропуске ударного слога на g-м икте: $\mathrm{z}=(\mathrm{k}-1) ; 1 \leq\left|\mathrm{U}_{\mathrm{Ci}}\right| \leq 2$, $\mathrm{i}=1,2, \ldots(\mathrm{k}-1)$ и $\mathrm{i} \neq(\mathrm{g}-1), 2 \leq\left|\mathrm{U}_{\mathrm{C}(\mathrm{g}-1)}\right| \leq 4 ; \quad\left|\mathrm{U}_{\mathrm{C}}\right|_{\max }=4, \quad\left|\mathrm{U}_{\mathrm{C}}\right|_{\min }=1 ; \quad\left|\mathrm{V}_{\mathrm{C}}\right|_{\max }=3, \quad\left|\mathrm{~V}_{\mathrm{C}}\right|_{\min }=0, \mathrm{v}_{\mathrm{C}}=3 ; \quad(\mathrm{k}-$ $1) \leq\left|\mathrm{W}_{\mathrm{C}}\right| \leq 2(\mathrm{k}-1), 2 \leq\left|\tilde{\mathrm{U}}_{\mathrm{C}}\right|=\left|\mathrm{W}_{\mathrm{C}}\right| /(\kappa-2) \leq 3$.

Для слова $\mathrm{P}_{\mathrm{Ci}}$ со слоговым объемом $\left|\mathrm{P}_{\mathrm{C}}\right|=\left(\mathrm{q}_{(\mathrm{i})}+\mathrm{h}_{(\mathrm{i})}+1\right) \geq 3$ имеет место ударение на икте. При $\mathrm{q}_{(\mathrm{i})} \geq 3$ или $\mathrm{h}_{(\mathrm{i})} \geq 3$ имеет место пропуск ударения на икте в предударной части или заударной части соответственно.

Объемные показатели при сверхсхемном ударении на g-м междуиктовом интервале: $\mathrm{z}=(\mathrm{k}+1) ; 1 \leq\left|\mathrm{U}_{\mathrm{Ci}}\right| \leq 2, \mathrm{i}=1,2, \ldots(\mathrm{k}+1)$ и $\mathrm{i} \neq \mathrm{g},(\mathrm{g}+1),\left|\mathrm{U}_{\mathrm{Cg}}\right|+\left.\mathrm{U}_{\mathrm{C}(\mathrm{g}+1)}|=2,| \mathrm{U}_{\mathrm{C}}\right|_{\max }=2,\left|\mathrm{U}_{\mathrm{C}}\right|_{\min }=1 ;\left|\mathrm{V}_{\mathrm{C}}\right|_{\max }=1$, $\left|\mathrm{V}_{\mathrm{C}}\right|_{\min }=0, \mathrm{v}_{\mathrm{C}}=1 ;(\mathrm{k}-1) \leq\left|\mathrm{W}_{\mathrm{C}}\right| \leq 2(\mathrm{k}-1),(\mathrm{k}-1) / \mathrm{k} \leq\left\lceil\tilde{\mathrm{U}}_{\mathrm{C}} \mid \leq\lceil 2(\mathrm{k}-1) / \mathrm{k}\rceil, 1 \leq\left\lceil\tilde{\mathrm{U}}_{\mathrm{C}} \mid \leq 2\right.\right.$.

Для слова $\mathrm{P}_{\mathrm{Ci}}$ со слоговым объемом $\left|\mathrm{P}_{\mathrm{Ci}}\right|=\left(\mathrm{q}_{(\mathrm{i})}+\mathrm{h}_{(\mathrm{i})}+1\right) \geq 2$ имеет место ударение на икте.

Объемные показатели при одновременном пропуске ударения на икте и сверхсхемном ударении: $\mathrm{z}=\mathrm{k} ; \quad\left|\mathrm{U}_{\mathrm{C}}\right|_{\max }=4, \quad\left|\mathrm{U}_{\mathrm{C}}\right|_{\min }=1 ; \quad\left|\mathrm{V}_{\mathrm{C}}\right|_{\max }=3, \quad\left|\mathrm{~V}_{\mathrm{C}}\right|_{\min }=0, \quad \mathrm{v}_{\mathrm{C}}=3 ; \quad(\mathrm{k}-1) \leq\left|\mathrm{W}_{\mathrm{C}}\right| \leq 2(\mathrm{k}-1)$; $1 \leq\left\lceil\tilde{\mathrm{U}}_{\mathrm{C}}|=| \mathrm{W}_{\mathrm{C}} \mid /(\mathrm{k}-1) \leq 2\right.$.

Для слова $\mathrm{P}_{\mathrm{Cj}}$ со слоговым объемом $\left|\mathrm{P}_{\mathrm{C}}\right|=\left(\mathrm{q}_{(\mathrm{i})}+\mathrm{h}_{(\mathrm{i})}+1\right) \geq 2$ имеет место ударение на икте. При $\mathrm{q}_{(\mathrm{i})} \geq 3$ или $\mathrm{h}_{(\mathrm{i})} \geq 3$ имеет место пропуск ударения на икте в предударной части или заударной части соответственно. 
Тактовики - метры с вариативностью объема междуиктовых интервалов $\mathrm{v}=2$, а для каждого вида метров $\mathrm{r}(1) \neq$ const, $\left|\mathrm{S}_{\mathrm{i}}\right| \neq$ const, $\left|\mathrm{V}_{\mathrm{i}}\right| \neq$ const, $|\mathrm{W}| \neq$ const, $\mathrm{s}_{\mathrm{A}}(\mathrm{k})=0,1,2$.

\section{Тактовик-1.}

Объемные показатели метрической схемы: $\mathrm{k} \geq 2 ; \mathrm{r}_{\mathrm{A}}(1) \leq 3 ; 2 \leq\left|\mathrm{U}_{\mathrm{Ai}}\right| \leq 4 ; 1 \leq\left|\mathrm{V}_{\mathrm{Ai}}\right| \leq 3 ; 2(\mathrm{k}-$ $1) \leq\left|\mathrm{W}_{\mathrm{A}}\right|=\sum_{\mathrm{i}=1,2, \ldots(\mathrm{k}-1)}\left|\mathrm{U}_{\mathrm{Ai}}\right| \leq 4(\mathrm{k}-1) ; 2 \leq\left|\tilde{\mathrm{U}}_{\mathrm{A}}\right| \leq 4$.

Объемные показатели при пропуске ударного слога на g-м икте: $\mathrm{z}=(\mathrm{k}-1) ; 2 \leq\left|\mathrm{U}_{\mathrm{Ci}}\right| \leq 4$, $\mathrm{i}=1,2, \ldots(\mathrm{k}-1)$ и $\mathrm{i} \neq(\mathrm{g}-1), 4 \leq\left|\mathrm{U}_{\mathrm{C}(\mathrm{g}-1)}\right| \leq 8 ;\left|\mathrm{U}_{\mathrm{C}}\right|_{\max }=8,\left|\mathrm{U}_{\mathrm{C}}\right|_{\min }=2 ;\left|\mathrm{V}_{\mathrm{C}}\right|_{\max }=7,\left|\mathrm{~V}_{\mathrm{C}}\right|_{\min }=1, \mathrm{v}_{\mathrm{C}}=6 ; 2(\mathrm{k}-$ $1) \leq\left|\mathrm{W}_{\mathrm{C}}\right| \leq 4(\mathrm{k}-1), 3 \leq\left|\tilde{\mathrm{U}}_{\mathrm{C}}\right|=\left|\mathrm{W}_{\mathrm{C}}\right| /(\mathrm{\kappa}-2) \leq 8$.

Для слова $\mathrm{P}_{\mathrm{Ci}}$ со слоговым объемом $\left|\mathrm{P}_{\mathrm{Ci}}\right|=\left(\mathrm{q}_{(\mathrm{i})}+\mathrm{h}_{(\mathrm{i})}+1\right) \geq 8$ имеет место ударение на икте. При $\mathrm{q}_{(\mathrm{i})} \geq 7$ или $\mathrm{h}_{(\mathrm{i})} \geq 7$ имеет место пропуск ударения на икте в предударной части или заударной части соответственно.

Объемные показатели при сверхсхемном ударении на g-м междуиктовом интервале: $\mathrm{z}=(\mathrm{k}+1) ; \quad 2 \leq\left|\mathrm{U}_{\mathrm{Ci}}\right| \leq 4, \quad \mathrm{i}=1,2, \ldots(\mathrm{k}+1) \quad$ и $\quad \mathrm{i} \neq \mathrm{g},(\mathrm{g}+1), \quad 2 \leq\left(\left|\mathrm{U}_{\mathrm{Cg}}\right|+\left.\mathrm{U}_{\mathrm{C}(\mathrm{g}+1)}|\leq 4, \quad| \mathrm{U}_{\mathrm{C}}\right|_{\max }=4, \quad\left|\mathrm{U}_{\mathrm{C}}\right|_{\min }=1 ;\right.$ $\left|\mathrm{V}_{\mathrm{C}}\right|_{\max }=3,\left|\mathrm{~V}_{\mathrm{C}}\right|_{\min }=0, \mathrm{v}_{\mathrm{C}}=3 ; 2(\mathrm{k}-1) \leq\left|\mathrm{W}_{\mathrm{C}}\right| \leq 4(\mathrm{k}-1),\left[2(\mathrm{k}-1) / \mathrm{k} \mid \leq\left\lceil\tilde{U}_{\mathrm{C}} \mid \leq\left[4(\mathrm{k}-1) / \mathrm{k} \mid, 2 \leq\left\lceil\tilde{U}_{\mathrm{C}} \mid \leq 4\right.\right.\right.\right.$.

Для слова $\mathrm{P}_{\mathrm{Cj}}$ со слоговым объемом $\left|\mathrm{P}_{\mathrm{Ci}}\right|=\left(\mathrm{q}_{(\mathrm{i})}+\mathrm{h}_{(\mathrm{i})}+1\right) \geq 4$ имеет место ударение на икте.

Объемные показатели при одновременном пропуске ударения на икте и сверхсхемном ударении: $\mathrm{z}=\mathrm{k} ; \quad\left|\mathrm{U}_{\mathrm{C}}\right|_{\max }=8, \quad\left|\mathrm{U}_{\mathrm{C}}\right|_{\min }=1 ; \quad\left|\mathrm{V}_{\mathrm{C}}\right|_{\max }=7, \quad\left|\mathrm{~V}_{\mathrm{C}}\right|_{\min }=0, \quad \mathrm{v}_{\mathrm{C}}=7 ; \quad 2(\mathrm{k}-1) \leq\left|\mathrm{W}_{\mathrm{C}}\right| \leq 4(\mathrm{k}-1)$; $2 \leq\left\lceil\tilde{U}_{\mathrm{C}}\right]=\left|\mathrm{W}_{\mathrm{C}}\right| /(\mathrm{k}-1) \leq 4$.

Для слова $\mathrm{P}_{\mathrm{Ci}}$ со слоговым объемом $\left|\mathrm{P}_{\mathrm{C}}\right|=\left(\mathrm{q}_{(\mathrm{i})}+\mathrm{h}_{(\mathrm{i})}+1\right) \geq 4$ имеет место ударение на икте. При $\mathrm{q}_{(\mathrm{i})} \geq 7$ или $\mathrm{h}_{(\mathrm{i})} \geq 7$ имеет место пропуск ударения на икте в предударной части или заударной части соответственно.

\section{Тактовик-2.}

Объемные показатели метрической схемы: $\mathrm{k} \geq 2 ; \mathrm{r}_{\mathrm{A}}(1) \leq 2 ; 1 \leq\left|\mathrm{U}_{\mathrm{Ai}}\right| \leq 3 ; 0 \leq\left|\mathrm{V}_{\mathrm{Ai}}\right| \leq 2 ; \quad$ (k$1) \leq\left|\mathrm{W}_{\mathrm{A}}\right|=\sum_{\mathrm{i}=1,2, \ldots(\mathrm{k}-1)}\left|\mathrm{U}_{\mathrm{Ai}}\right| \leq 3(\mathrm{k}-1) ; 1 \leq\left|\tilde{\mathrm{U}}_{\mathrm{A}}\right| \leq 3$.

Объемные показатели при пропуске ударного слога на g-м икте: $\mathrm{z}=(\mathrm{k}-1) ; 1 \leq\left|\mathrm{U}_{\mathrm{Ci}}\right| \leq 3$, $\mathrm{i}=1,2, \ldots(\mathrm{k}-1)$ и $\mathrm{i} \neq(\mathrm{g}-1), 2 \leq\left|\mathrm{U}_{\mathrm{C}(\mathrm{g}-1)}\right| \leq 6 ;\left|\mathrm{U}_{\mathrm{C}}\right|_{\max }=6, \quad\left|\mathrm{U}_{\mathrm{C}}\right|_{\min }=1 ;\left|\mathrm{V}_{\mathrm{C}}\right|_{\max }=5, \quad\left|\mathrm{~V}_{\mathrm{C}}\right|_{\min }=0, \mathrm{v}_{\mathrm{C}}=5 ; 2(\mathrm{k}-$ $1) \leq\left|\mathrm{W}_{\mathrm{C}}\right| \leq 3(\mathrm{k}-1), 3 \leq\left|\tilde{\mathrm{U}}_{\mathrm{C}}\right|=\left|\mathrm{W}_{\mathrm{C}}\right| /(\kappa-2) \leq 6$.

Для слова $\mathrm{P}_{\mathrm{Ci}}$ со слоговым объемом $\left|\mathrm{P}_{\mathrm{C}}\right|=\left(\mathrm{q}_{(\mathrm{i})}+\mathrm{h}_{(\mathrm{i})}+1\right) \geq 6$ имеет место ударение на икте. При $\mathrm{q}_{(\mathrm{i})} \geq 5$ или $\mathrm{h}_{(\mathrm{i})} \geq 5$ имеет место пропуск ударения на икте в предударной части или заударной части соответственно.

Объемные показатели при сверхсхемном ударении на g-м междуиктовом интервале: $\mathrm{z}=(\mathrm{k}+1) ; \quad 1 \leq\left|\mathrm{U}_{\mathrm{Ci}}\right| \leq 3, \quad \mathrm{i}=1,2, \ldots(\mathrm{k}+1) \quad$ и $\quad \mathrm{i} \neq \mathrm{g},(\mathrm{g}+1), \quad 1 \leq\left(\left|\mathrm{U}_{\mathrm{Cg}}\right|+\left.\mathrm{U}_{\mathrm{C}(\mathrm{g}+1)}|\leq 3, \quad| \mathrm{U}_{\mathrm{C}}\right|_{\max }=3, \quad\left|\mathrm{U}_{\mathrm{C}}\right|_{\min }=1 ;\right.$ $\left|\mathrm{V}_{\mathrm{C}}\right|_{\max }=2,\left|\mathrm{~V}_{\mathrm{C}}\right|_{\min }=0, \mathrm{v}_{\mathrm{C}}=2 ;(\mathrm{k}-1) \leq\left|\mathrm{W}_{\mathrm{C}}\right| \leq 3(\mathrm{k}-1),\left[(\mathrm{k}-1) / \mathrm{k} \mid \leq\left\lceil\tilde{\mathrm{U}}_{\mathrm{C}}|\leq\lceil 3(\mathrm{k}-1) / \mathrm{k}\rceil, 1 \leq| \tilde{\mathrm{U}}_{\mathrm{C}}\right] \leq 3\right.$.

Для слова $\mathrm{P}_{\mathrm{Ci}}$ со слоговым объемом $\left|\mathrm{P}_{\mathrm{Ci}}\right|=\left(\mathrm{q}_{(\mathrm{i})}+\mathrm{h}_{(\mathrm{i})}+1\right) \geq 3$ имеет место ударение на икте.

Объемные показатели при одновременном пропуске ударения на икте и сверхсхемном ударении: $\mathrm{z}=\mathrm{k} ; \quad\left|\mathrm{U}_{\mathrm{C}}\right|_{\max }=6, \quad\left|\mathrm{U}_{\mathrm{C}}\right|_{\min }=1 ; \quad\left|\mathrm{V}_{\mathrm{C}}\right|_{\max }=5, \quad\left|\mathrm{~V}_{\mathrm{C}}\right|_{\min }=0, \quad \mathrm{v}_{\mathrm{C}}=5 ; \quad(\mathrm{k}-1) \leq\left|\mathrm{W}_{\mathrm{C}}\right| \leq 3(\mathrm{k}-1) ;$ $1 \leq\left|\tilde{\mathrm{U}}_{\mathrm{C}}\right|=\left|\mathrm{W}_{\mathrm{C}}\right| /(\mathrm{k}-1) \leq 3$.

Для слова $\mathrm{P}_{\mathrm{Ci}}$ со слоговым объемом $\left|\mathrm{P}_{\mathrm{Ci}}\right|=\left(\mathrm{q}_{(\mathrm{i})}+\mathrm{h}_{(\mathrm{i})}+1\right) \geq 3$ имеет место ударение на икте. При $\mathrm{q}_{(\mathrm{i})} \geq 5$ или $\mathrm{h}_{(\mathrm{i})} \geq 5$ имеет место пропуск ударения на икте в предударной части или заударной части соответственно.

Ударник (урегулированный акцентный или чисто тонический стих).

Объемные показатели метрической схемы: $\mathrm{k} \geq 2 ; \quad \mathrm{r}(1) \leq 8 ; \quad 1 \leq\left|\mathrm{U}_{\mathrm{i}}\right| \leq 9 ; \quad 0 \leq\left|\mathrm{V}_{\mathrm{i}}\right| \leq 8 ; \quad \mathrm{v} \geq 3$, $\mathrm{k} \leq|\mathrm{W}|=\left(1+\sum_{\mathrm{i}=1,2, \ldots(\mathrm{k}-1)}\left|\mathrm{U}_{\mathrm{i}}\right|\right) \leq(9 \mathrm{k}-8) ; \mathrm{s}(\mathrm{k})=0,1,2$.

Такие метры сильно расшатаны и рассматривать отклонения от метра нет смысла, поскольку урегулированность метра утрачивается.

Дисметрический стих (не урегулированный акцентный стих).

Стих без метрической схемы: $\mathrm{k} \neq$ const, $\mathrm{r}(1) \neq$ const, $\left|\mathrm{U}_{\mathrm{i}}\right| \neq$ const, $\left|\mathrm{V}_{\mathrm{i}}\right| \neq$ const, $\mathrm{v} \neq$ const, $|\mathrm{W}| \neq$ const.

Верлибр - не рифмованный дисметрический стих.

Раешный стих - рифмованный дисметрический стих.

Единственный объемный показатель - рифма с клаузулами $\mathrm{s}(\mathrm{k})=0,1,2$. 
Сегментный стих из урегулированных частей, силлабо-тонических и тонических, при не урегулированном сочленении здесь не рассматривается.

\section{3. Равносложные метры}

Метры с равносложными строками - строгие метры при k=const. К их числу, относятся такие равносложные метры как логаэд и силлабический стих. Отличие силлабических стихов от равносложных силлабо-тонических определяется наличием у последних анакрус равного объема.

Логаэдический стих может иметь в своей основе смешанные размеры силлаботонического и тонического метров с равным объемом сопоставляемых строк $(\mathrm{r}(1)+|\mathrm{W}|+\mathrm{s}(\mathrm{k}))$ и при полном совпадении фиксированных регулятивов: $\mathrm{k} ; \mathrm{r}(1) ;\left|\mathrm{U}_{\mathrm{i}}\right| ;\left|\mathrm{V}_{\mathrm{i}}\right| ;|\mathrm{W}|$; $\mathrm{s}(\mathrm{k})=0,1,2$.

Силлабический стих не имеет в своей основе урегулированных метров и содержит лишь такие регулятивы: равные объемы сопоставляемых строк $|\mathrm{F}|=(\mathrm{r}(1)+|\mathrm{W}|+\mathrm{s}(\mathrm{k}))$ и женских клаузул $\mathrm{s}(\mathrm{k})=1$.

Различают две формы силлабического стиха:

- бесцезурный $|\mathrm{F}|$-сложный силлабический стих с объемом строки $7 \leq|\mathrm{F}| \leq 10$;

- цезурированный $|\mathrm{F}|$-сложный силлабический стих с объемом строки $11 \leq|\mathrm{F}| \leq 16$ и с цезурой (с фиксированным словоразделом) после 5-го слога, т.е. регулятивом $\left|\mathrm{F}_{\mathrm{i}}\right| \geq 5$.

\section{5. Реализация процедур автоматической идентификации метра}

Формально-языковая модель русского стиха была алгоритмизирована и был разработан программный комплекс, позволяющий проводить спецификацию поэтического произведения.

Адрес ресурса: https://wikipoetics.ru

Программный код автоматического анализа стиха будет выложен в свободный доступ: https://github.com/mariakaryaeva/Poetics

\section{1. Основные ресурсы}

\section{ТЕХНИЧЕСКИЕ СРЕДСТВА}

Программный код написан на высокоуровневом языке Python. B проекте использовались открытые библиотеки (руMystem, pymorphy2, numpy, scikit-learn), позволяющие проводить обработку русского языка. Так как реализация данного функционала носит прикладной характер, то для создания удобного интерфейса для пользователей был создан web-сайт с использованием фреймворка Django.

ПРОГРАММНАЯ СТРУКТУРА

класс СТИХ

raw_verse - стиховой текст в первоначальном виде без обработки

list_of_lines - список строк типа СТРОКА

класс СТРОКА

raw_line - строка в первоначальном виде без обработки

text_only_line - строка, состоящая из набора слов из raw_line, разделенных пробелом.

Любые символы (кроме русского алфавита) отсутствуют

by_words - список слов, содержащийся в строке

syllables_number - сумма всех слогов в строке

rythm_lines - словарь со списком рифмующихся строк (ключ), ранг сходства строк (значение)

stress_schema - схема ударений в строке (формат: cCCcccC, где C - ударный слог, с безударный слог)

phonetich_words - список фонетических слов в строке 
anakruz - анакруса

clause - клаузула

класс СЛОВО

raw_word - слово в первоначальном виде без обработки

norm_word - первоначальное слово, которое поступило на вход. Без регуляции регистра, нормализации (начальная форма)

stress_word - слово с ударением по словарю Зализняка

stress_word_probability - вероятность ударения в атрибуте stress_word

probab_stress_list - словарь, в котором собраны всевозможные простановки ударения для слова (ключи) и их вероятности (значения). Формируется путем комбинации расстановки ударения поочередно на все гласные

POS - часть речи слова

shemaCc - схема ударения в слове для stress_word (формат: сCсc, где C - ударный слог, c - безударный слог)

\section{2. Этапы алгоритмизации}

Далее будут кратко и последовательно описаны основные этапы алгоритма спецификации поэтического текста с более подробным описанием значимых процедур.

1 ЭТАП

Для каждого стихового текста происходит разделение на строки с использованием символа конца строки и выполняется заполнение структуры класса СТИХ.

Очистка строк от символов, отличных от символов русского алфавита и пробела.

Разделение строк на слова.

2 ЭТАП

Для каждой строки выполняется заполнение структуры класса СТРОКА. Схема ударений и разделение на метрические слова происходит на следующем этапе.

3 ЭТАП

Для каждого слова выполняется процедура поиска ударения по словарю Зализняка [9]. Если ударение не определяется однозначно или вовсе не определяется по словарю Зализняка, то выполняется процедура формирования вероятностных ударений. Кроме того, если ударение однозначно может быть определено по словарю Зализняка, то возможен случай, когда данное слово теряет ударение в силу наличия ритма стиха или может быть изменено по соображениям автора поэтического текста. Таким образом, при наличии единственного ударения, вероятность определяется неоднозначно.

ПРОЦЕДУРА ВЕРОЯТНОСТНОЙ АКЦЕНТУАЦИИ:

Подсчет количества слогов в слове $(|\mathrm{S}|)$

Исходя из количества слогов:

$|\mathrm{S}|=1$ :

- Часть речи - частица, то $\mathrm{p}=1 / 2$

- Иначе, $\mathrm{p}=1$

$|\mathrm{S}|=2$ :

- В словаре единственный вариант: $p=3 / 4$ ( $1 / 4-$ остаток, что может быть авторский перенос ударения )

- В словаре оба варианта: вероятность каждого $\mathrm{p}=1 / 2$

- В словаре нет вариантов: вероятность каждого $\mathrm{p}=1 / 2$

$|\mathrm{S}|>=3$, где $\mathrm{N}$ - количество гласных, $\mathrm{M}$ - количество возможных ударений из словаря Зализняка:

— Вероятность для слова, ударение которого есть в словаре $=1 / \mathrm{N}+1 /(\mathrm{N} * \mathrm{~N})$

- Вероятность для слова, ударения которого нет в словаре $=\left(1-\mathrm{M}^{*}(1 / \mathrm{N}+\right.$ $1 /(\mathrm{N} * \mathrm{~N}))) /(\mathrm{N}-\mathrm{M})$

Добавка $1 /(\mathrm{N} * \mathrm{~N})$ позволяет заведомо увеличить вероятность для слова с ударением из словаря Зализняка по сравнению предполагаемым словом-кандидатом. Слова-кандидаты 
могут быть двух типов: ударение было перенесено специально по задумке автора или ударение не отражено в словаре Зализняка по каким-либо причинам (новое слово, транслитерация иностранного слова и т.д.).

КОНЕЦ ПРОЦЕДУРЫ.

Для каждого слова выполняется заполнение структуры класса СЛОВО. Часть речи и нормальная форма слова определяется с помощью библиотеки pymorphy2 (https://pymorphy2.readthedocs.io/).

\section{4 ЭТАП}

Для каждой строки выполняется процедура поиска ритмических слов. Данная процедура использует множества препозитивов и постпозитивов.

ПРОЦЕДУРА ПОИСКА ФОНЕТИЧЕСКИХ СЛОВ:

1. В строке, длиной $\mathrm{k}$ слов, последовательно рассматриваются слова, начиная с 1 до (k-

1). Текущее слово обозначим (c_i) и последующее слово (n_i). Множество препозитивов PRE, множество постпозитивов POST, слова, не принадлежащие, ни множеству PRE, ни множеству POST, относим к множеству WORD:

1.1. rythm_word $=[] / /$ пустое фонетическое слово

1.2. $c \_i$ in PRE and $n \_i$ in WORD $\Rightarrow>$ add $c \_i$ to rythm_word

1.3. $c \_i$ in PRE and $n \_i$ in PRE $\Rightarrow$ add $c \_i$ to rythm_word

1.4. $c \_i$ in POST and $n \_i$ in WORD $\Rightarrow>$ add $c \_i$ to rythm_word, next rythm_word $=[]$

1.5. $c \_i$ in POST and $n \_i$ in PRE $\Rightarrow>$ add $c \_i$ to rythm_word, next rythm_word $=[]$

1.6. $c_{-}$i in POST and $n \_i$ in POST $\Rightarrow>$ add $c \_i$ to rythm_word

1.7. $c \_i$ in WORD and $n \_i$ in WORD $\Rightarrow>$ add $c \_i$ to rythm_word, next rythm_word $=[]$

1.8. $\mathrm{c} \_\mathrm{i}$ in WORD and $\mathrm{n} \_\mathrm{i}$ in POST $=>$ add $\mathrm{c} \_\mathrm{i}$ to rythm_word

1.9. $c \_i$ in WORD and $n \_i$ in PRE $=>$ add $c \_i$ to rythm_word, next rythm_word $=[]$

2. Для k-ого слова:

2.1. add c_i to rythm_word

КОНЕЦ ПРОЦЕДУРЫ.

После полного формирования структуры стиха происходит работа уже над вычислением характеристик стиха. Для этого используются полученные на этапе формирования структуры стиха промежуточные результаты. К основным промежуточным результатам, которые будут служить основой для определения полной спецификации стиха, можно отнести: вероятностная схема ударений в словах, метрические слова.

\section{5 ЭТАП}

В рамках данного уровня происходит переход к абстракции стиха в целом.

Первоочередной задачей является поиск рифмы между строками [6].

ПРОЦЕДУРА ПОИСКА РИФМ:

1. Для каждой строки извлекается последнее фонетическое слово каждой строки.

2. Для каждого фонетического слова выполняется определение заударной части и предударной части.

3. Для каждой пары фонетических слов выполняется поиск схем созвучий согласно разработанным схемам и проставляется ранг (оценка созвучности).

4. Для каждой строки выбирается строки с оценками созвучности выше Q (задается в настройках), в случае, если количество пар, определившихся как созвучные больше чем одна.

\section{КОНЕЦ ПРОЦЕДУРЫ.}

Процедуры вычисления схем достаточно просты за счет формирования классов и атрибутов классов, поскольку необходимо получить для каждой строки ударную схему слова. Соединив значения необходимых атрибутов по словам/фонетическим словам/строкам, получаем общую схему стиха.

\section{ПРОЦЕДУРА ВЫЧИСЛЕНИЯ СЛОГОВОЙ СХЕМЫ}

1. Для каждой строки, для каждого слова извлекается ударная схема слова.

2. Соединение результата для каждого слова в строки. 
КОНЕЦ ПРОЦЕДУРЫ.

ПРОЦЕДУРА ВЫЧИСЛЕНИЯ СХЕМЫ СЛОВОРАЗДЕЛОВ

1. Для каждой строки извлекаются фонетические слова.

2. Для каждого слова в фонетическом слове извлекается количество слогов.

3. Для каждого слова значение количества слогов последовательно записывается от 1 до номера количества слогов (пример строки: 1s 234s 56s 78910).

4. Соединение результата фонетических слов в строки.

КОНЕЦ ПРОЦЕДУРЫ.

ПРОЦЕДУРА ИДЕНТИФИКАЦИИ СТИХА

1. Для каждой строки вычисление значений регулятивов: метрического периода или стопы $|\mathrm{Si}|$, междуиктового интервала $|\mathrm{Vi}|$, анакрусы r(1), клаузулы s(k), основы стиха $|\mathrm{W}|$, подцепочек до словораздела после i-го икта $|\mathrm{Fi}|$

2. Для каждой строки сопоставление значений регулятивов формулам для определения размера стропы

3. Для каждой строки определение метра стопы по длине анакрусы

КОНЕЦ ПРОЦЕДУРЫ

ДОПОЛНИТЕЛЬНАЯ ПРОЦЕДУРА (итеративНЫй Подход)

Для увеличения качества по точности был введен дополнительный итеративный подход, который основывается на переборе вероятностей ударений в слове и наложении заранее сгенерированных метрических схем на полные слоговые схемы ударений. В таком случае, ударный слог обозначается 1 , а безударный 0 . В конечном итоге получаем набор матриц, состоящих из 0 и 1 . Отдельно генерируется по каждому метрическому размеру схема и сопоставляется с матрицами стиха. Максимальное совпадение метрической схемы и схемы стиха показывает тип метра стиха.

\section{6. Заключение}

Для решения поставленной задачи была рассмотрена формально-языковая модель слогового представления стиха и регулятивов его метра. В этой модели были выделены конституирующие как метр, так и ритм метро-ритмические сегменты строки стихового текста, слоговые объемы которых задают комплекс регулятивов его метра. Было привлечено определение метра русского стиха, сформулированное в [13], опору которому составили такие измеримые сегменты стиха как фонетичское слово и метрический период с междуиктовым интервалом.

В решении задачи идентификации метра стихового текста следует выделить два последовательных этапа.

На первом этапе выявлено наличие рифмы и на основе таких регулятивов, как объем основы стиха, анакрусы и клаузулы, разрешена альтернатива выбора из систематического перечня системы стихосложения, которой принадлежит исследуемый стиховой текст.

На втором этапе введен такой показатель как регулятор метра, связанный со средним значением объема метро-ритмического периода и вычисляемый для каждого метра выбранной для исследуемого текста системы стихосложения.

При рассмотрении из репертуара выпали такие метры тонической системы стихосложения как сегментный и былинный стих, которые стиховедчески исследованы еще не в достаточной мере.

В заключительной процедуре на основе определения метра стиха производится:

- для выявления ударного слога на икте сравнение указанного регулятора с объемами фонетических слов,

- для выявления пропуска ударения на икте сравнение их предударных и заударных сегментов фонетических слов с максимальной величиной междуакцентного интервала в строке.

В завершающей главе описаны процедуры автоматической идентификации метра. 
Работа поддержана Российским фондом фундаментальных исследований, гранты № 1606-00497 и № 16-07-01180.

\section{Литература}

[1] Аншаков О.М. Проблема автоматического определения метра и ритма русского стиха: математические модели и алгоритмы // Труды Института русского языка им. В. В. Виноградова. М., 2017. Вып. 11: Славянский стих. С. 179-188.

[2] Ахманова О.С. Группа акцентная // Словарь лингвистических терминов. М., 1966. C. 117.

[3] Барахнин В.Б., Кожемякина О.Ю. Об автоматизации комплексного анализа русского поэтического текста // CEUR Workshop Proceedings. 2012. Vol. 934. P. 167-171.

[4] Бойков В.Н. Контекстно-свободная грамматика одной ритмической модели русского стиха // МАИС. 2012. Т. 19, № 4. С. 154-167.

[5] Бойков В.Н., Каряева М.С., Соколов В.А., Пильщиков И.А. Об автоматической спецификации стиха в информационно-аналитической системе // CEUR Workshop Proceedings. 2015. Vol. 1536. P. 144-151.

[6] Бойков В.Н., Каряева М.С., Пильщиков И.А. Формально-языковая модель рифмующихся слов для автоматического поиска // Компьютерная лингвистика и вычислительные онтологии. (Труды XXI Международной объединенной конференции «Интернет и современное общество, IMS-2018, Санкт-Петербург, 30 мая - 2 июня 2018 г.). 2018. № 2. С. 25-36.

[7] Гаспаров М.Л. Современный русский стих: Метрика и ритмика. М., 1974.

[8] Гаспаров М.Л. Русский стих начала XX века в комментариях. М., 2001.

[9] Зализняк А.А. Грамматический словарь русского языка. Словоизменение. М., 1980.

[10] Зализняк А.А. Труды по акцентологии. М., 2010. Т. І. С. 118-121, 817-826.

[11] Козьмин А.В. Автоматический анализ стиха в системе STARLING // Компьютерная лингвистика и интеллектуальные технологии: Труды международной конференции «Диалог 2006» (Бекасово, 31 мая - 4 июня 2006 г.). М., 2006. С. 265-268.

[12] Пильщиков И.А. Безударные икты и сверхсхемные ударения в русском акцентном стихе: «По ту сторону дольника». Доклад на Гаспаровских чтениях - 2017. Москва, РГГУ, 20 апреля 2017 г. URL: https:/www.academia.edu/32577333/ (дата обращения: 29.04.2019).

[13] Пильщиков И.А. Понятия стих, метр и ритм в русской стиховедческой традиции // Труды Института русского языка им. В.В. Виноградова. М., 2017. Вып. 11: Славянский стих. С. 12-30.

[14] Пильщиков И.А., Старостин А. С. Проблема автоматического распознавания метра: силлаботоника, дольник, тактовик // Отечественное стиховедение: 100-летние итоги и перспективы развития: Материалы Международной научной конференции 25-27 ноября 2010 г. Санкт-Петербург / под ред. С. И. Богданова, Е. В. Хворостьяновой. СПб.: Филологический факультет СПбГУ, 2010. С. 397-406.

[15] Скулачева Т.В. Методы определения метра в неклассическом стихе // Изв. РАН. Сер. лит. и яз. 2012. № 2. С. 42-55.

[16] Томашевский Б.В. Русское стихосложение. Метрика. Пг., 1923. (Вопросы поэтики; Вып. II).

[17] Торсуева И.Г. Ритм // Лингвистический энциклопедический словарь. М., 1990. С. 416. 
[18] Pilshchikov I., Starostin A. Automated Analysis of Poetic Texts and the Problem of Verse Meter // Current Trends in Metrical Analysis / C. Küper (ed.). Bern; Berlin; [etc.]: Peter Lang. 2011. Vol. 2. P. 133-140.

[19] Pilshchikov I., Starostin A. Reconnaissance automatique des mètres des vers russes: une approche statistique sur corpus // Langages. 2015. № 199. P. 89-105.

\title{
A Formal Language Model of Verse and the Regulatives of Verse Meters for Automated Meter Recognition
}

\author{
V. Boikov ${ }^{1}$, M. Kariaeva ${ }^{1}$, I. Pilshchikov ${ }^{2,3}$ \\ ${ }^{1}$ Yaroslavl Demidov State University, ${ }^{2}$ Lomonosov Moscow State University, ${ }^{3}$ Tallinn \\ University
}

The paper presents a formal language model of the syllable representation of the poetic text and regulatives of verse meters, determined by the parameters of particular segments of a line.

Metro-rhythmical segments are identified that constitute both the meter and the rhythm of verse. A definition of a meter of Russian verse is used, which is supported by such measurable segments of the verse as the phonetic word and the meter-generating verse period with its interictic interval.

The values of regulatives for the repertoire of meters of Russian verse are determined. Such phenomena as the extra-schematic stresses and skipping of schematic stresses are also considered.

The procedures for automatic recognition of verse meters are described.

Keywords: verse, verse segmentation, syllabic volume of a verse segment, inter-ictic interval, meter-generating verse period, verse meter, verse meter regulatives

Reference for citation: Boikov V.N., Kariaeva M.S., Pilshchikov I.A. A Formal Language Model of Verse and the Regulatives of Verse Meters for Automated Meter Recognition // Computer Linguistics and Computing Ontologies. Vol. 3 (Proceedings of the XXII International Joint Scientific Conference «Internet and Modern Society», IMS-2019, St. Petersburg, June 1922, 2019). - St. Petersburg: ITMO University, 2019. P. 124 - 141. DOI: 10.17586/2541-97812019-3-124-141

\section{Reference}

[1] Anshakov O.M. Problema avtomaticheskogo opredeleniya metra i ritma russkogo stikha: matematicheskiye modeli i algoritmy // Trudy Instituta russkogo yazyka im. V. V. Vinogradova. M., 2017. Vyp. 11: Slavyanskiy stikh. P. 179-188.

[2] Akhmanova O.S. Gruppa aktsentnaya // Slovar' lingvisticheskikh terminov. M., 1966.P. 117.

[3] Barakhnin V.B., Kozhemyakina O.YU. Ob avtomatizatsii kompleksnogo analiza russkogo poeticheskogo teksta // CEUR Workshop Proceedings. 2012. Vol. 934. P. 167-171.

[4] Boykov V.N. Kontekstno-svobodnaya grammatika odnoy ritmicheskoy modeli russkogo stikha // MAIS. 2012. T. 19, № 4. P. 154-167.

[5] Boykov V.N., Karyayeva M.S., Sokolov V.A., Pil'shchikov I.A. Ob avtomaticheskoy spetsifikatsii stikha $\mathrm{v}$ informatsionno-analiticheskoy sisteme // CEUR Workshop Proceedings. 2015. Vol. 1536. P. 144-151. 
[6] Boykov V.N., Karyayeva M.S., Pil'shchikov I.A. Formal'no-yazykovaya model' rifmuyushchikhsya slov dlya avtomaticheskogo poiska // Komp'yuternaya lingvistika i vychislitel'nyye ontologii. 2018. № 2. P. 25-36.

[7] Gasparov M.L. Sovremennyy russkiy stikh: Metrika i ritmika. M., 1974.

[8] Gasparov M.L. Russkiy stikh nachala KHKH veka v kommentariyakh. M., 2001.

[9] Zaliznyak A.A. Grammaticheskiy slovar' russkogo yazyka. Slovoizmeneniye. M., 1980.

[10]Zaliznyak A.A. Trudy po aktsentologii. M., 2010. T. I. P. 118-121, 817-826.

[11] Koz'min A.V. Avtomaticheskiy analiz stikha v sisteme STARLING // Komp'yuternaya lingvistika i intellektual'nyye tekhnologii: Trudy mezhdunarodnoy konferentsii «Dialog 2006» (Bekasovo, 31 maya - 4 iyunya 2006 g.). M., 2006. P. 265-268.

[12] Pil'shchikov I.A. Bezudarnyye ikty i sverkhskhemnyye udareniya v russkom aktsentnom stikhe: «Po tu storonu dol'nika». Doklad na Gasparovskikh chteniyakh - 2017. Moskva, RGGU, 20 aprelya 2017 g. URL: https://www.academia.edu/32577333/ (data obrashcheniya: 29.04.2019).

[13] Pil'shchikov I.A. Ponyatiya stikh, metr i ritm v russkoy stikhovedcheskoy traditsii // Trudy Instituta russkogo yazyka im. V.V. Vinogradova. M., 2017. Vol. 11: Slavyanskiy stikh. P. 12-30.

[14] Pil'shchikov I.A., Starostin A. S. Problema avtomaticheskogo raspoznavaniya metra: sillabotonika, dol'nik, taktovik // Otechestvennoye stikhovedeniye: 100-letniye itogi i perspektivy razvitiya: Materialy Mezhdunarodnoy nauchnoy konferentsii 25-27 noyabrya 2010 g. Sankt-Peterburg / pod red. S. I. Bogdanova, Ye. V. Khvorost'yanovoy. SPb.: Filologicheskiy fakul'tet SPbGU, 2010. P. 397-406.

[15] Skulacheva T.V. Metody opredeleniya metra v neklassicheskom stikhe // Izv. RAN. Ser. lit. i yaz. 2012. № 2. P. 42-55.

[16] Tomashevskiy B.V. Russkoye stikhoslozheniye. Metrika. Pg., 1923. (Voprosy poetiki; Vol. II).

[17] Torsuyeva I.G. Ritm // Lingvisticheskiy entsiklopedicheskiy slovar'. M., 1990. P. 416.

[18]Pilshchikov I., Starostin A. Automated Analysis of Poetic Texts and the Problem of Verse Meter // Current Trends in Metrical Analysis / C. Küper (ed.). Bern; Berlin; [etc.]: Peter Lang, 2011. P. 133-140. (Littera: Studies in Language and Literature; Vol. 2).

[19]Pilshchikov I., Starostin A. Reconnaissance automatique des mètres des vers russes: une approche statistique sur corpus // Langages. 2015. № 199. P. 89-105. 